\title{
The complete far-infrared and submillimeter spectrum of the Class 0 protostar Serpens SMM1 obtained with Herschel
}

\section{Characterizing UV-irradiated shocks heating and chemistry $\star, \star \star$}

\author{
J. R. Goicoechea ${ }^{1}$, J. Cernicharo ${ }^{1}$, A. Karska ${ }^{2,3}$, G. J. Herczeg ${ }^{4}$, E. T. Polehampton ${ }^{5,6}$, \\ S. F. Wampfler ${ }^{7}$, L. E. Kristensen ${ }^{3}$, E. F. van Dishoeck ${ }^{2,3}$, M. Etxaluze ${ }^{1}$, O. Berné ${ }^{8,9}$, and R. Visser ${ }^{10}$ \\ ${ }^{1}$ Centro de Astrobiología (CSIC/INTA), Ctra. de Torrejón a Ajalvir, km 4, 28850 Torrejón de Ardoz, Madrid, Spain \\ e-mail: jr.goicoechea@cab.inta-csic.es \\ 2 Max-Planck-Institut für extraterrestrische Physik (MPE), Postfach 1312, 85741 Garching, Germany \\ ${ }^{3}$ Leiden Observatory, Leiden University, PO Box 9513, 2300 RA Leiden, The Netherlands \\ ${ }^{4}$ Kavli Institute for Astronomy and Astrophysics, Peking University, Yi He Yuan Lu 5, HaiDian Qu, 100871 Beijing, PR China \\ 5 RAL Space, Rutherford Appleton Laboratory, Chilton, Didcot, Oxfordshire, OX11 0QX, UK \\ 6 Institute for Space Imaging Science, University of Lethbridge, 4401 University Drive, Lethbridge, Alberta T1J 1B1, Canada \\ 7 Centre for Star and Planet Formation, Natural History Museum of Denmark, University of Copenhagen, Øster Voldgade5-7, \\ 1350 København K, Denmark \\ ${ }^{8}$ Université de Toulouse, UPS-OMP, IRAP, Toulouse, France \\ 9 CNRS, IRAP, 9 Av. colonel Roche, BP 44346, 31028 Toulouse Cedex 4, France \\ 10 Department of Astronomy, University of Michigan, 500 Church St., Ann Arbor, MI 48109, USA
}

Received 28 June 2012 / Accepted 17 September 2012

\begin{abstract}
We present the first complete $\sim 55-671 \mu$ m spectral scan of a low-mass Class 0 protostar (Serpens SMM1) taken with the PACS and SPIRE spectrometers onboard Herschel. More than 145 lines have been detected, most of them rotationally excited lines of ${ }^{12} \mathrm{CO}$ (full ladder from $J_{\mathrm{u}}=4-3$ to $42-41$ and $E_{\mathrm{u}} / k=4971 \mathrm{~K}$ ), $\mathrm{H}_{2} \mathrm{O}$ (up to $8_{18}-7_{07}$ and $E_{\mathrm{u}} / k=1036 \mathrm{~K}$ ), OH (up to ${ }^{2} \Pi_{1 / 2} J=7 / 2-5 / 2$ and $E_{\mathrm{u}} / k=618 \mathrm{~K}$ ), ${ }^{13} \mathrm{CO}$ (up to $J_{\mathrm{u}}=16-15$ ), $\mathrm{HCN}$ and $\mathrm{HCO}^{+}$(up to $J_{\mathrm{u}}=12-11$ ). Bright [O I]63, $145 \mu \mathrm{m}$ and weaker [C II] 158 and $\left[\mathrm{C}\right.$ I] 370, $609 \mu \mathrm{m}$ lines are also detected, but excited lines from chemically related species $\left(\mathrm{NH}_{3}, \mathrm{CH}^{+}, \mathrm{CO}^{+}, \mathrm{OH}^{+}\right.$or $\left.\mathrm{H}_{2} \mathrm{O}^{+}\right)$are not. Mid-infrared spectra retrieved from the Spitzer archive are also first discussed here. The $\sim 10-37 \mu \mathrm{m}$ spectrum has many fewer lines, but shows clear detections of [Ne II], [Fe II], [Si II] and [S I] fine structure lines, as well as weaker $\mathrm{H}_{2} \mathrm{~S}(1)$ and $\mathrm{S}(2)$ pure rotational lines. The observed line luminosity is dominated by $\mathrm{CO}(\sim 54 \%), \mathrm{H}_{2} \mathrm{O}(\sim 22 \%)$, [O I] ( 12\%) and $\mathrm{OH}(\sim 9 \%)$ emission. A multi-component radiative transfer model allowed us to approximately quantify the contribution of the three different temperature components suggested by the ${ }^{12} \mathrm{CO}$ rotational ladder $\left(T_{\mathrm{k}}^{\text {hot }} \approx 800 \mathrm{~K}, T_{\mathrm{k}}^{\text {warm }} \approx 375 \mathrm{~K}\right.$ and $\left.T_{\mathrm{k}}^{\text {cool }} \approx 150 \mathrm{~K}\right)$. Gas densities $n\left(\mathrm{H}_{2}\right) \approx 5 \times 10^{6} \mathrm{~cm}^{-3}$ are needed to reproduce the observed far-IR lines arising from shocks in the inner protostellar envelope (warm and hot components) for which we derive upper limit abundances of $x(\mathrm{CO}) \lesssim 10^{-4}, x\left(\mathrm{H}_{2} \mathrm{O}\right) \lesssim 0.2 \times 10^{-5}$ and $x(\mathrm{OH}) \lesssim 10^{-6}$ with respect to $\mathrm{H}_{2}$. The lower energy submm ${ }^{12} \mathrm{CO}$ and $\mathrm{H}_{2} \mathrm{O}$ lines show more extended emission that we associate with the cool entrained outflow gas. Fast dissociative $J-$ shocks $\left(v_{\mathrm{s}}>60 \mathrm{~km} \mathrm{~s}^{-1}\right)$ within an embedded atomic jet, as well as lower velocity small-scale non-dissociative shocks $\left(v_{\mathrm{s}} \lesssim 20 \mathrm{~km} \mathrm{~s}^{-1}\right)$ are needed to explain both the atomic fine structure lines and the hot $\mathrm{CO}$ and $\mathrm{H}_{2} \mathrm{O}$ lines respectively. Observations also show the signature of $\mathrm{UV}$ radiation (weak [C II] and [C I] lines and high $\mathrm{HCO}^{+} / \mathrm{HCN}$ abundance ratios) and thus, most observed species likely arise in UV-irradiated shocks. Dissociative $J$-shocks produced by a jet impacting the ambient material are the most probable origin of $[\mathrm{O} \mathrm{I}]$ and $\mathrm{OH}$ emission and of a significant fraction of the warm $\mathrm{CO}$ emission. In addition, $\mathrm{H}_{2} \mathrm{O}$ photodissociation in UV-irradiated non-dissociative shocks along the outflow cavity walls can also contribute to the [O I] and $\mathrm{OH}$ emission.
\end{abstract}

Key words. stars: protostars - ISM: jets and outflows - infrared: ISM - shock waves

\section{Introduction}

In the earliest stages of evolution, low-mass protostars are deeply embedded in dense envelopes of molecular gas and dust. During collapse, conservation of angular momentum combined with infall along the magnetic field lines leads to the formation of a

\footnotetext{
* Herschel is an ESA space observatory with science instruments provided by European-led Principal Investigator consortia and with important participation from NASA.

$\star \star$ Appendix $\mathrm{A}$ is available in electronic form at

http://www . aanda.org
}

dense rotating protoplanetary disk that drives the accretion process. At the same time, both mass and angular momentum are removed from the system by the onset of jets, collimated flows, and the magnetic braking action (e.g., Bontemps et al. 1996; Bachiller \& Tafalla 1999). The resulting outflow produces a cavity in the natal envelope with walls subjected to energetic shocks and strong radiation fields from the protostar. These processes heat the circumstellar gas and leave their signature in the prevailing chemistry (e.g., enhanced abundances of water vapour).

Both the line and continuum emission from young stellar objects (YSOs) peak in the far-infrared (far-IR) and thus they are 
robust diagnostics of the energetic processes associated with the first stages of star formation (e.g., Giannini et al. 2001). The improved sensitivity and angular/spectral resolution of Herschel spectrometers compared to previous far-IR observatories allows us to detect a larger number of far-IR lines and to better constrain their spatial origin. This is especially true for the detection of high excitation, optically thin lines that can help us to identify high temperature components as well as to disentangle and quantify the dominant heating mechanisms (mechanical vs. radiative). Early Herschel observations of a few lowmass protostars for which partial or complete PACS spectra are available show that the molecular emission of relatively excited ${ }^{12} \mathrm{CO}(J<30), \mathrm{H}_{2} \mathrm{O}$ and $\mathrm{OH}$ lines is a common feature (van Kempen et al. 2010 for HH46; and Benedettini et al. 2012 for $\mathrm{L} 1157-\mathrm{B} 1$ ). This warm $\mathrm{CO}$ and $\mathrm{H}_{2} \mathrm{O}$ emission was suggested to arise from the walls of an outflow-carved cavity in the envelope, which are heated by UV photons and by non-dissociative $C$-type shocks (van Kempen et al. 2010; Visser et al. 2012). The [OI] and $\mathrm{OH}$ emission observed in these sources, however, was proposed to arise from dissociative $J$-shocks. Herczeg et al. (2012) detected higher- $J{ }^{12} \mathrm{CO}$ lines (up to $J=49-48$ ) and highly excited $\mathrm{H}_{2} \mathrm{O}$ lines in the NGC 1333 IRAS 4B outflow indicating the presence of even hotter gas. The very weak [O I] $63 \mu \mathrm{m}$ line emission in IRAS 4B outflow (the [O I] $145 \mu \mathrm{m}$ line is not even detected) led these authors to conclude that the hot gas where $\mathrm{H}_{2} \mathrm{O}$ dominates the gas cooling is heated by nondissociative $C$-shocks shielded from UV radiation. Passive heating by the protostellar luminosity is also thought to contribute to the mid- $J{ }^{12} \mathrm{CO}$ and ${ }^{13} \mathrm{CO}$ emission (Visser et al. 2012; Y1ld1z et al. 2012). In NGC 1333 IRAS 4A/4B, however, the ${ }^{12} \mathrm{CO}$ intensities and broad line-profiles of lower- $J$ transitions $(J=1-0$ up to 10-9) probe swept-up or entrained shocked gas along the outflow (Y1ldiz et al. 2012).

In this paper we present the first complete far-IR and submm spectra of a Class 0 protostar (Serpens SMM1 or FIRS 1), taken with the PACS (Poglitsch et al. 2010) and SPIRE (Griffin et al. 2010) spectrometers onboard the Herschel Space Observatory (Pilbratt et al. 2010). SMM1 is a low-mass protostellar system located in the Serpens core (Eiroa et al. 2008) at a distance of $d=230 \pm 20$ pc (see also Dzib et al. 2010, for an alternative measurement). It is the most massive low-mass Class 0 source $\left(M_{\mathrm{env}} \simeq 16.1 M_{\odot}, T_{\mathrm{bol}} \simeq 39 \mathrm{~K}\right)$ of the WISH program sample (Water in Star-forming Regions with Herschel, van Dishoeck et al. 2011). The Herschel spectra are complemented with midIR Spitzer Infrared Spectrograph (IRS) archive spectra of the embedded protostar (photometry first presented by Enoch et al. 2009).

The Serpens cloud core was first mapped in the far-IR with the LWS spectrometer onboard the Infrared Space Observatory (ISO). Even with a poor angular and spectral resolution of $\sim 80^{\prime \prime}$ and $R=\lambda / \Delta \lambda \sim 200$, ISO-LWS observations of Serpens SMM1 revealed a rich spectrum of molecular lines (Larsson et al. 2002), superposed onto a strong far-IR continuum (Larsson et al. 2000). In particular, ${ }^{12} \mathrm{CO}$ (up to $J=21-20$ ), $\mathrm{H}_{2} \mathrm{O}$ (up to $4_{41}-3_{30}$ ) and some $\mathrm{OH}$ lines were detected. Detailed modeling of the ISO emission concluded that those species trace the inner $\sim 10^{3}$ AU regions around the protostar, where gas temperatures are above $300 \mathrm{~K}$ and densities above $10^{6} \mathrm{~cm}^{-3}$. The comparison of the $\mathrm{H}_{2} \mathrm{O}, \mathrm{OH}$ and $\mathrm{CO}$ lines with existing shock models suggested that shock heating along the SMM1 outflow is the most likely mechanism of molecular excitation. The details of these shocks, however, were less clear.

Because of its far-IR luminosity and rich spectrum, Serpens SMM1 is an ideal Class 0 source for studying the protostellar environment and to characterize the shock- and UV-heating and chemistry with the much higher spatial and spectral capabilities provided by Herschel. In the following sections we present the data set, we identify the observed lines, present a 3 component non-local thermodynamic equilibrium (non-LTE) radiative transfer model and compare the observations with available shock models and observations of other YSOs.

\section{Observations and data reduction}

\subsection{Herschel PACS and SPIRE observations}

PACS spectra between $\sim 55$ and $\sim 210 \mu \mathrm{m}$ were obtained on 31 October 2010 in the range spectroscopy mode. The PACS spectrometer uses photoconductor detectors and provides 25 spectra over a $47^{\prime \prime} \times 47^{\prime \prime}$ field-of-view $(\mathrm{FoV})$ resolved in $5 \times 5$ spatial pixels ("spaxels"), each with a size of $\sim 9.4$ " on the sky. The resolving power varies between $R \sim 1000$ (at $\sim 100 \mu \mathrm{m}$ in the R1 grating order) and $\sim 5000$ (at $\sim 70 \mu \mathrm{m}$ in the B3A order). The central spaxel was centered at the Serpens SMM1 protostar $\left(\alpha_{2000}: 18^{\mathrm{h}} 29^{\mathrm{m}} 49.8^{\mathrm{s}}, \delta_{2000}: 1^{\circ} 15^{\prime} 20.5^{\prime \prime}\right)$. Observations were carried out in the "chop-nodded" mode with the largest chopper throw of 6 arcmin. The total observing time was $\sim 3 \mathrm{~h}$ (observation IDs 1342207780 and 1342207781). The measured width of the spectrometer point spread function (PSF) is relatively constant for $\lambda \lesssim 100 \mu \mathrm{m}(F W H M \simeq$ spaxel size) but increases at longer wavelengths. In particular only $\simeq 40 \%(\simeq 70 \%)$ of a point source emission would fall in the central spaxel at $\simeq 190 \mu \mathrm{m}(\lambda \simeq 60 \mu \mathrm{m})$. Hence, extracting line surface brightness from a semi-extended source like Serpens SMM1 is not trivial. In this work we followed the method described by Herczeg et al. (2012) and Karska et al. (2012). This involves multiplying all line fluxes measured in the central spaxel with a $\lambda$-dependent correction curve calculated from the extended emission of the brightest $\mathrm{CO}$ and $\mathrm{H}_{2} \mathrm{O}$ lines observed over the entire FoV. The correction factor is constant $(\sim 2)$ for $\lambda<120 \mu \mathrm{m}$ and increases from $\sim 2$ to $\sim 3-4$ at $\sim 200 \mu \mathrm{m}$. For the continuum and [O I] and [C II] lines, the individual line fluxes measured in all spaxels were added. Note that we checked that the reference spectra in the two off positions are free of [C II] emission to confirm that the $[\mathrm{C} \mathrm{II}]$ lines towards Serpens SMM1 are real. Figure 1 shows the PACS spectrum in the central spaxel and Fig. 2 shows the entire PACS array from 55 to $190 \mu \mathrm{m}$ (the bright emission line is $[\mathrm{O} \mathrm{I}] 63 \mu \mathrm{m})$.

SPIRE FTS spectra between $\sim 194$ and $\sim 671 \mu \mathrm{m}$ (1545-447 GHz) were obtained on 27 March 2011. The SPIRE FTS uses two bolometer arrays covering the 194-313 $\mu \mathrm{m}$ (short wavelength array, SSW) and 303-671 $\mu \mathrm{m}$ (long wavelength array, SLW) bands. The two arrays contain 19 (SLW) and 37 (SSW) hexagonally packed detectors separated by $\sim 2$ beams (51' and 33" respectively). The unvignetted FoV is $\sim 2^{\prime}$. The observation was centered at Serpens SMM1 (IDs 1342216893) in the high spectral resolution mode $\left(\Delta \tilde{\lambda}=0.04 \mathrm{~cm}^{-1}\right.$; $R \sim 500-1000)$. The total integration time was $\sim 9 \mathrm{~min}$. We corrected the spectrum to account for the extended continuum and molecular emission of the source and the changing beam size with frequency across the SPIRE band. Taking account of the coupling of the source to the SPIRE-FTS beam (Wu et al., in prep.) and using the overlap region between the SSW and SLW bands as a reference (where the beam size differs by a factor of $\sim 2$ ) we calculate the FWHM size of the submm continuum source to be $\sim 20^{\prime \prime}$. The SPIRE continuum fluxes were appropriately corrected and the unapodized spectrum was used to fit the line intensities with sinc functions. Four 
J. R. Goicoechea et al.: The complete spectrum of the Class 0 protostar Serpens SMM1

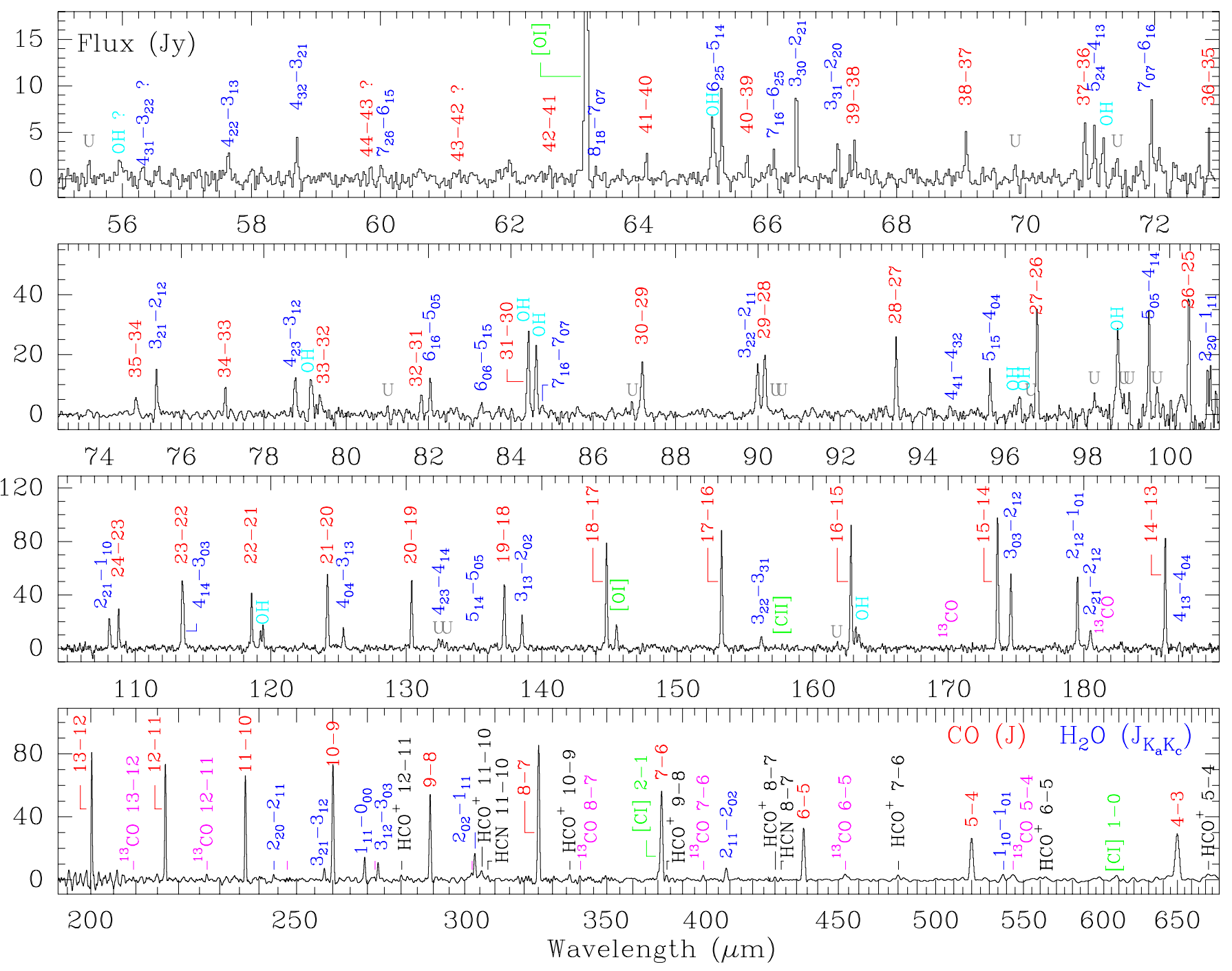

Fig. 1. PACS and SPIRE (lower panel) continuum-subtracted spectra of Serpens SMM1 (central spaxel) with $\mathrm{H}_{2} \mathrm{O}$ (blue), $\mathrm{CO}$ (red), $\mathrm{OH}$ (cyan), ${ }^{13} \mathrm{CO}$ (magenta), [O I], [C II] and [C I] (green), and $\mathrm{HCO}^{+}$and $\mathrm{HCN}$ (black) lines labelled. The flux density scale is in Jy (assuming a point source).

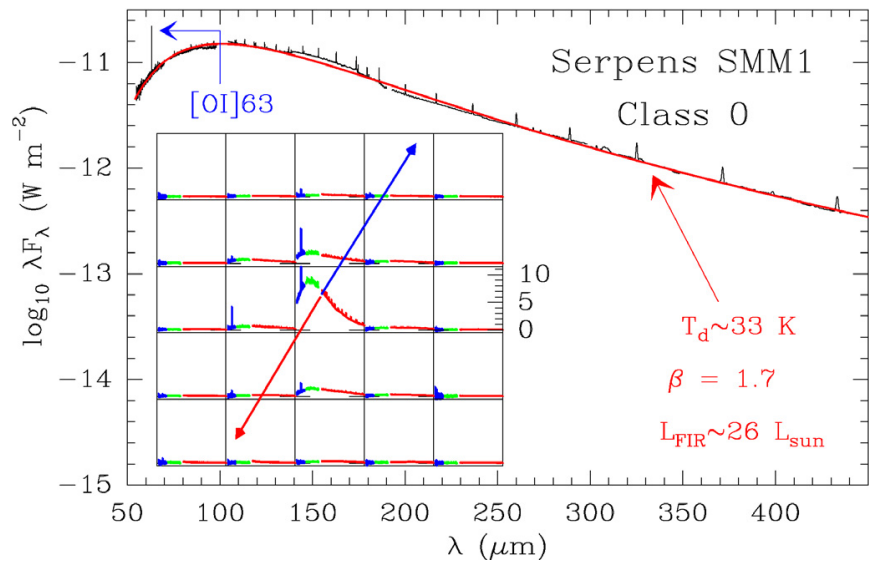

Fig. 2. Observed spectral energy distribution (SED) of Serpens SMM1 and modified blackbody fit (red curve). Inset shows the full PACS array in flux density units of $10^{-14} \mathrm{~W} \mathrm{~m}^{-2} \mu \mathrm{m}^{-1}$. The abscissa is in linear scale from 55 to $190 \mu \mathrm{m}$. The bright emission line seen in several positions is [O I] $63 \mu \mathrm{m}$. Approximate outflow directions are shown with red and blue arrows.

representative mid- $J{ }^{12} \mathrm{CO}$ and $\mathrm{H}_{2} \mathrm{O}$ integrated line intensity maps are shown in Fig. 3.
SPIRE and PACS data were processed using HIPE and then exported to GILDAS where basic line spectrum manipulations were carried out. Table A.1 summarize the detected lines and the line fluxes towards the protostar position (central spaxel; corrected for extended emission in the case of PACS observations). The spatial distribution of several atomic and molecular lines observed in the PACS array is shown in Fig. 4.

\subsection{Spitzer IRS Observations}

In order to complement the far-IR and submm Herschel spectra we have also analyzed mid-IR data towards the protostar position from the Infrared Spectrograph (IRS) onboard Spitzer (Houck et al. 2004) in high spectral resolution mode $(R \sim 600$; 9.9-37.2 $\mu \mathrm{m}$ ). The basic calibrated data (BCD) files for the short high (SH) and long high ( $\mathrm{LH})$ orders of IRS were retrieved from the Spitzer Heritage Archive ${ }^{1}$ (OBSID 34330, PI M. Enoch). Pipelining of the data was achieved using CUBISM (Smith et al. 2007) to assemble the BCD files into spectral cubes. Bad and rogue pixels were removed using 3-sigma filtering. The spectra

\footnotetext{
1 http://sha.ipac.caltech.edu/applications/Spitzer/ SHA/
} 

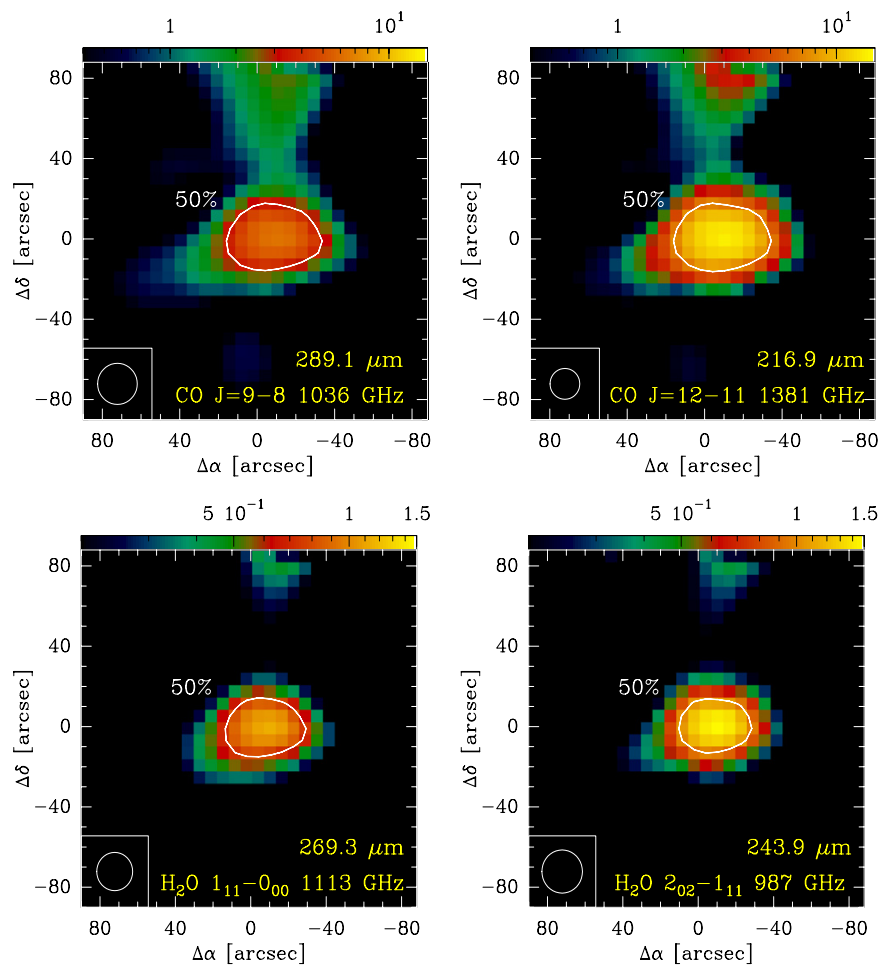

Fig. 3. Upper: SPIRE-FTS ${ }^{12} \mathrm{CO} J=9-8$ and $12-11$ sparse maps Lower: $p-\mathrm{H}_{2} \mathrm{O} 1_{11}-0_{00}$ and $2_{02}-1_{11}$ maps respectively. The line surface brightness is on the $10^{-8} \mathrm{~W} \mathrm{~m}^{-2} \mathrm{sr}^{-1}$ scale. The white contours represent the $50 \%$ line emission peak level. The FWHM beam is shown in each inset. Note the increase of line surface brightness in the more excited lines.

were extracted in a $\sim 7^{\prime \prime} \times 7^{\prime \prime}$ aperture, thus close to the PACS spaxel size. The resulting Spitzer IRS spectra are shown in Fig. 5. The photometry of these data was first presented by Enoch et al. (2009). Table A.2 summarizes the detected lines and their fluxes.

\section{Results}

\subsection{Far-infrared and submillimeter lines}

Figure 1 shows the very rich PACS and SPIRE (lower panel) spectrum of the Serpens SMM1 protostar. More than 145 lines have been detected, most of them rotationally excited lines from abundant molecules: $38{ }^{12} \mathrm{CO}$ lines (up to $J=42-41$ and $E_{\mathrm{u}} / k=4971 \mathrm{~K}$ ), 37 lines of both $o-\mathrm{H}_{2} \mathrm{O}$ and $p-\mathrm{H}_{2} \mathrm{O}$ (up to $8_{18}-7_{17}$ and $E_{\mathrm{u}} / k=1036 \mathrm{~K}$ ), $16 \mathrm{OH}$ lines (up to ${ }^{2} \Pi_{1 / 2} J=$ $7 / 2-5 / 2$ and $E_{\mathrm{u}} / k=618 \mathrm{~K}$ ), $12{ }^{13} \mathrm{CO}$ lines (up to $J=16-15$ and $E_{\mathrm{u}} / k=719 \mathrm{~K}$ ) and several $\mathrm{HCN}$ and $\mathrm{HCO}^{+}$lines (up to $J=$ $12-11$ and $E_{\mathrm{u}} / k=283 \mathrm{~K}$ ). Weaker [C II] $158 \mu \mathrm{m}$ and [C I]370, $609 \mu \mathrm{m}$ lines are also detected. Excited lines from $\mathrm{NH}_{3}, \mathrm{CH}^{+}$, $\mathrm{CO}^{+}, \mathrm{OH}^{+}$or $\mathrm{H}_{2} \mathrm{O}^{+}$are however not detected at the PACS and SPIRE spectral resolutions and sensitivity. The brightest line in the spectra is the [OI]63 $\mu \mathrm{m}$ line (see also Fig. 2) with a luminosity of $L_{63} \simeq 0.014 L_{\odot}$. Assuming no extinction in the far-IR, we measure $[\mathrm{OI}] 63 \mu \mathrm{m} /[\mathrm{C} \mathrm{II}] 158 \mu \mathrm{m} \simeq 30$ and $[\mathrm{OI}] 63 \mu \mathrm{m} /[\mathrm{O} \mathrm{I}] 145 \mu \mathrm{m} \simeq 10$ line flux ratios integrated over the entire PACS array.

Most of the far-IR continuum and line emission arises from the protostar position (central spaxel) with weaker, but detectable, contributions from adjacent outflow positions (see Fig. 4). In particular, the high excitation lines of $\mathrm{CO}, \mathrm{H}_{2} \mathrm{O}$ and $\mathrm{OH}$ detected below $\lambda<100 \mu \mathrm{m}$ seem to show a more compact distribution than the lower excitation lines (within the PACS PSF sampling caveats). The highest PACS spectral resolution $\left(\sim 90-60 \mathrm{~km} \mathrm{~s}^{-1}\right)$ is achieved in the $\sim 60-70 \mu \mathrm{m}$ range. Although at this resolution all detected molecular lines are spectrally unresolved (they have narrower intrinsic line-widths), the observed [O I] $63 \mu \mathrm{m}$ line is $>30 \%$ broader than any close-by molecular line in the $\sim 60-70 \mu \mathrm{m}$ range. This likely indicates that PACS marginally resolves the [O I] $63 \mu \mathrm{m}$ line, being broader (or having higher velocity line-wing emission) than the excited far-IR CO, $\mathrm{H}_{2} \mathrm{O}$ and $\mathrm{OH}$ lines. In addition, the $[\mathrm{O} \mathrm{I}] 63 \mu \mathrm{m}$ line-profile peak shifts in velocity from the protostar position (where the line is brightest) to the outflow red-lobe position where the line peak is redshifted by $\sim 100 \mathrm{~km} \mathrm{~s}^{-1}$ (more than a resolution element) and the molecular line emission is weaker (see red boxes in Fig. 4). Note that $[\mathrm{C}$ II $] 158 \mu \mathrm{m}$ peaks at this red-lobe position and the line peak is also redshifted by $\sim 100 \mathrm{~km} \mathrm{~s}^{-1}$ (although less than a resolution element in this wavelength range). Similar [O I]63 $\mu \mathrm{m}$ velocity-shifts are also seen in sources such as HH46 and are associated with the emission from an atomic jet itself (van Kempen et al. 2010). We refer to Karska et al. (2012) for a detailed comparison of the [O I] $63 \mu \mathrm{m}$ shifts in several outflows.

Finally, the lower energy submm lines, from ${ }^{12} \mathrm{CO}$ and $\mathrm{H}_{2} \mathrm{O}$ in particular, show a more extended distribution than the far-IR lines, with some indication of outflow emission in the the S-E direction (see SPIRE maps in Fig. 3).

Table 1 summarizes the total line luminosities adding all observed lines between $\sim 55$ and $671 \mu \mathrm{m}$. Note that a few unidentified lines (U) are present in the PACS spectrum.

\subsection{Mid-infrared lines and extinction}

The mid-IR spectrum of Serpens SMM1 (Fig. 5a) shows a much less rich spectrum than in the far-IR domain (probably due to severe dust extinction and lack of spectral resolution). Nevertheless, two weak $\mathrm{H}_{2}$ pure rotational lines (0-0 S(1) and $S(2)$ transitions) and seven brighter atomic fine-structure lines of $\mathrm{Ne}^{+}, \mathrm{Si}^{+}, \mathrm{S}$ and $\mathrm{Fe}^{+}$(four transitions) are clearly detected $^{2}$ at the protostar position (Figs. 5b and c). In contrast with NGC 1333-IRAS 4B (Watson et al. 2007), no mid-IR OH and $\mathrm{H}_{2} \mathrm{O}$ lines are seen. The presence of [Ne II] and [Fe II] lines from energy levels above a few thousands Kelvin, together with the bright and velocity-shifted [O I] line detected by Herschel, suggests the presence of fast dissociative shocks close to the protostar (e.g., Hollenbach \& McKee 1989; Neufeld \& Dalgarno 1989). Note that the same mid-IR lines are readily detected in Herbig-Haro objects and outflow lobes (e.g., Neufeld et al. 2006; Melnick et al. 2008). As discussed in Sect. 4, the observed intensities of $\mathrm{H}_{2}, \mathrm{H}_{2} \mathrm{O}$ and $\mathrm{CO}$ rotational lines are more typical of slower velocity shocks.

The large amount of gas and dust in embedded YSOs requires to apply extinction corrections to retrieve corrected midIR line luminosities. In this work we have taken grain optical properties from Laor \& Draine (1993) and computed an extinction curve $A(\lambda) / A_{V}$ from IR to submm wavelengths characterized by $R_{V}=A_{V} / E(B-V)=5.5$ (the ratio of visual extinction to reddening) and a conversion factor from extinction to hydrogen column density of $1.4 \times 10^{21} \mathrm{~cm}^{-2} \mathrm{mag}^{-1}$. These values have been previously used to correct photometric observations of embedded YSOs in the Serpens cloud (Evans et al. 2009). Table 1 lists the uncorrected mid-IR line luminosities and

2 First ionization potential of observed ionic species are: $21.56(\mathrm{Ne})$, $11.26(\mathrm{C}), 10.36(\mathrm{~S}), 8.15(\mathrm{Si})$ and $7.90 \mathrm{eV}(\mathrm{Fe})$. 

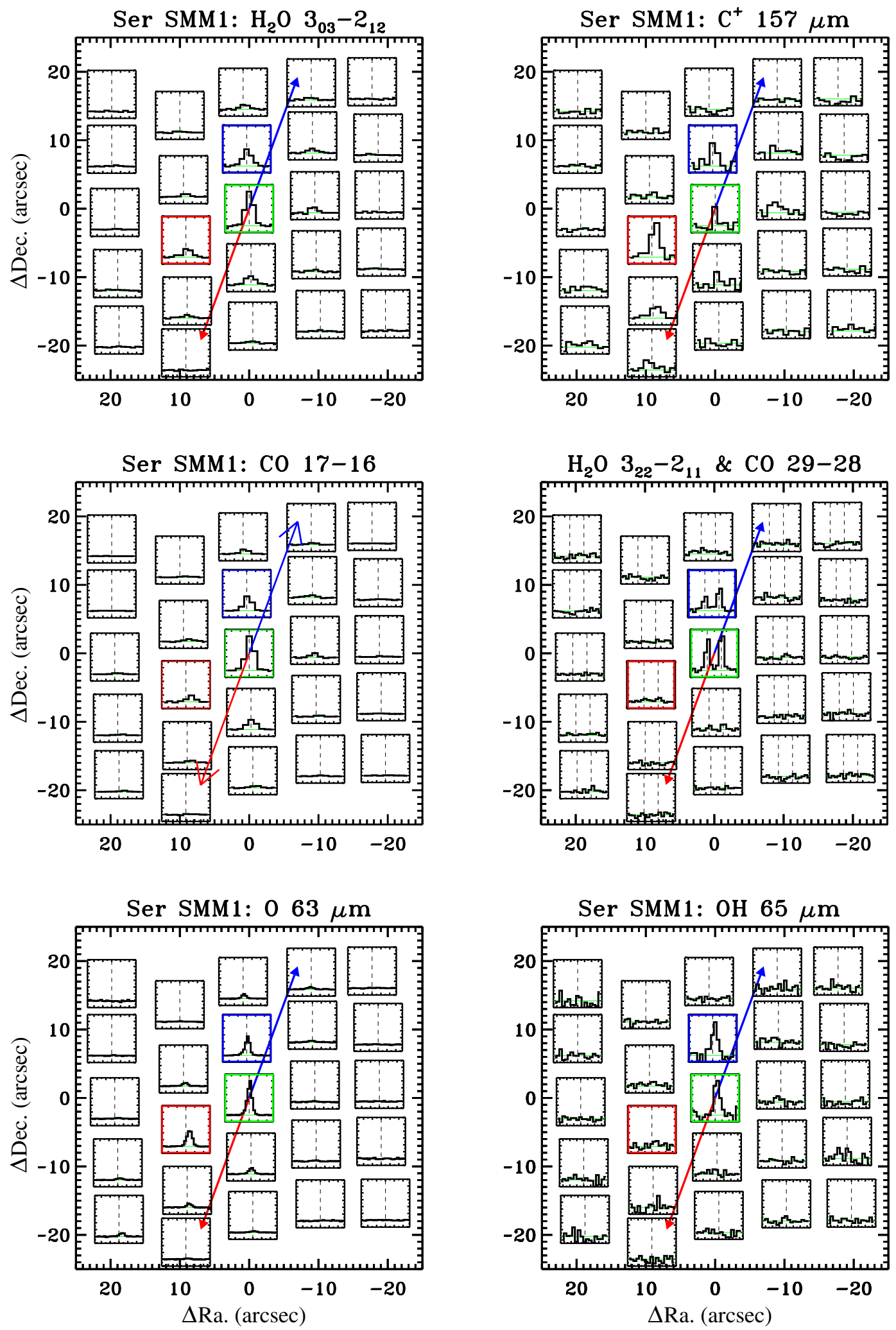

Fig. 4. PACS spectral maps of Serpens SMM1 in the $o-\mathrm{H}_{2} \mathrm{O} 3_{03}-2_{12}(174.626 \mu \mathrm{m})$; [C II] $157.741 \mu \mathrm{m}$; CO $J=17-16(153.267 \mu \mathrm{m})$; both $p-\mathrm{H}_{2} \mathrm{O} 3_{22}-2_{11}(89.988 \mu \mathrm{m})$ and CO $J=29-28(90.163 \mu \mathrm{m}) ;[\mathrm{O} \mathrm{I}] 63.183 \mu \mathrm{m}$ and $\mathrm{OH}^{2} \Pi_{3 / 2} J=9 / 2^{+}-7 / 2^{-}(65.279 \mu \mathrm{m})$ lines (from top to bottom). The center of each spaxel corresponds to its offset position with respect to the protostar at $\alpha_{2000}: 18^{\mathrm{h}} 29^{\mathrm{m}} 49.8^{\mathrm{s}}, \delta_{2000}: 1^{\circ} 15^{\prime} 20.5^{\prime \prime}$. The $Y$-axis for each transition represents the normalized line flux (in the -0.2 to 1.2 range) with respect to the brightest line in the array. The $X$-axis represents the -550 to $550 \mathrm{~km} \mathrm{~s}^{-1}$ velocity scale except for the $\mathrm{OH} 65.279 \mu \mathrm{m}$ line $\left(-350\right.$ to $\left.350 \mathrm{~km} \mathrm{~s}^{-1}\right)$ and the $p$ - $\mathrm{H}_{2} \mathrm{O} 89.988 \mu \mathrm{m}$ and $\mathrm{CO} 90.163 \mu \mathrm{m} \mathrm{lines}$ $\left(-1000\right.$ to $\left.1000 \mathrm{~km} \mathrm{~s}^{-1}\right)$. The vertical dashed lines show the position of the rest wavelengths. Blue and red arrows show the approximated outflow direction. The green box represents the central spaxel centered at the protostar position whereas the red and blue boxes represent postions in the red and blue outflow lobes respectively. Note that the PSF is relatively constant for $\lambda \lesssim 100 \mu \mathrm{m}\left(F W H M \simeq\right.$ spaxel size $\left.\simeq 9.4^{\prime \prime}\right)$ but increases at longer wavelengths. 

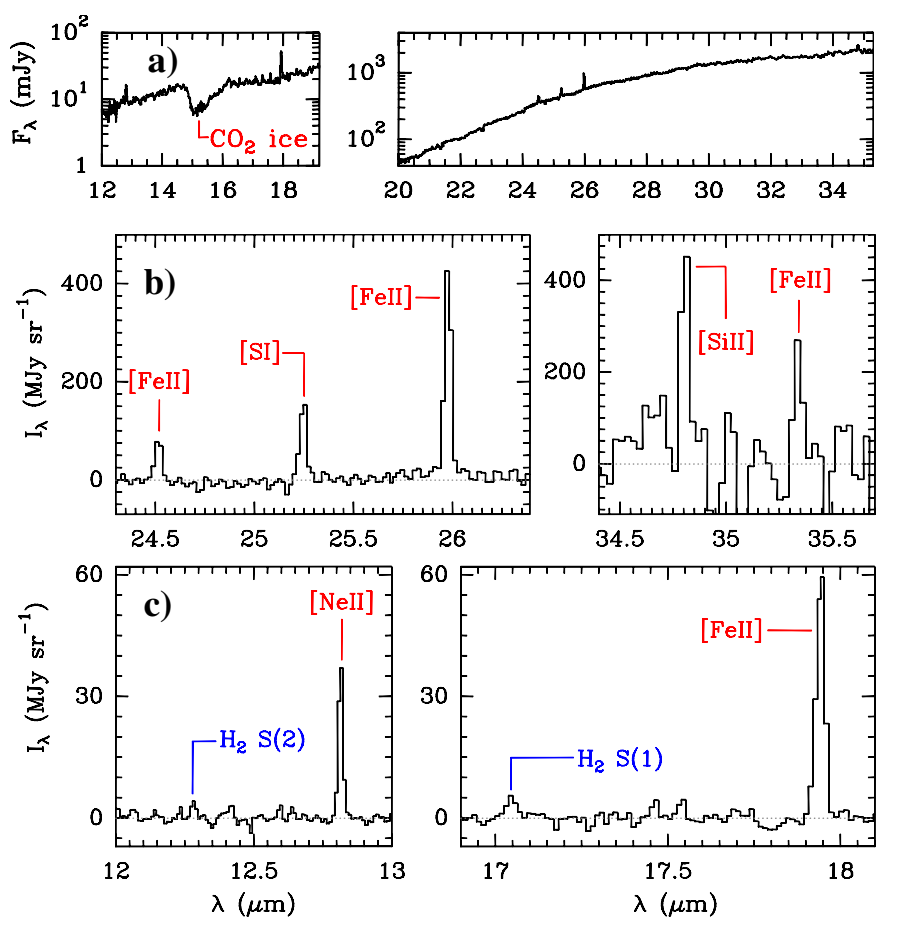

Fig. 5. a) Spitzer IRS spectrum of the Class 0 protostar Serpens SMM1 using the Hi Res modules and a 7" $\times 7$ " aperture. b) Continuumsubtracted spectra with main narrow lines identified in the LH module $(\lambda \geq 19.5 \mu \mathrm{m})$ and $\mathbf{c})$ with the SH module $(\lambda \leq 19.5 \mu \mathrm{m})$. Note the lack of bright molecular line emission $\left(\mathrm{H}_{2} \mathrm{O}, \mathrm{OH}\right.$, etc. $)$.

Table 1. Observed and modelled luminosities.

\begin{tabular}{lccc}
\hline \hline Species & $\log _{10} L_{\text {obs }}\left(L_{\odot}\right)$ & $\%^{a}$ & $\%$ of $L_{\text {obs }}$ in each component \\
& \\
${ }^{12} \mathrm{CO}$ & -1.17 & $54 \%$ & $\sim 15 \%(h) \sim 60 \%(w) \sim 25 \%(c)$ \\
$\mathrm{H}_{2} \mathrm{O}$ & -1.56 & $22 \%$ & $\sim 90 \%(h) \sim 10 \%(c)$ \\
{$[\mathrm{O} \mathrm{I}]$} & -1.82 & $12 \%$ & $\sim 100 \%(w)$ \\
$\mathrm{OH}$ & -1.94 & $9 \%$ & $\sim 100 \%(w)$ \\
${ }^{13} \mathrm{CO}$ & -2.75 & $1 \%$ & $\sim 40 \%(w) \sim 60 \%(c)$ \\
{$[\mathrm{C} \mathrm{II}]$} & -3.32 & $0 \%$ & $\sim 100 \%(w)$ \\
{$[\mathrm{C} \mathrm{I}]$} & -3.42 & $0 \%$ & $\lesssim 10 \%(w) \gtrsim 90 \%(c)$ \\
Dust & 1.41 & - & \\
\hline $\mathrm{H}_{2}$ & $-\left(4.83-2.54^{\dagger}\right)$ & & \\
{$[\mathrm{Fe}$ II $]$} & $-\left(3.57-2.62^{\dagger}\right)$ & & \\
{$[\mathrm{Si}$ II $]$} & $-\left(3.98-2.03^{\dagger}\right)$ & \\
{$[\mathrm{S} \mathrm{I}]$} & $-\left(4.52-3.07^{\dagger}\right)$ & \\
{$[\mathrm{Ne}$ II $]$} & $-\left(4.93-2.67^{\dagger}\right)$ & \\
\hline
\end{tabular}

Notes. ${ }^{(a)}$ Fraction of total far-IR/submm line luminosity. ${ }^{(b)}$ From simple model: $h=$ hot, $w=$ warm, $c=$ cool component. ${ }^{(\dagger)}$ From Spitzer IRS observations $(10-37 \mu \mathrm{m})$. Upper limits include an arbitrary extinction correction of $A_{V}=150 \mathrm{mag}$.

those using an arbitrary correction of $A_{V}=150$. In the latter case, even the $[\mathrm{OI}] 63 \mu \mathrm{m}$ line would be affected by extinction (the de-reddened line flux would be $\sim 2$ times higher) and the [O I $] 63 / 145 \mu \mathrm{m}$ line flux ratio would increase to $\sim 20$. Such large intensity ratios are predicted by dissociative shocks models (e.g., Hollenbach \& McKee 1989; Neufeld \& Dalgarno 1989; Flower $\&$ Pineau Des Forêts 2010) and thus provide a reasonable upper value to the extinction in the inner envelope.

An independent estimate of the extinction is obtained from the $\sim 9.7 \mu \mathrm{m}$ silicate grains absorption band seen in the Spitzer IRS low resolution spectra (shown in Enoch et al. 2009). In the diffuse ISM, the optical depth of the $\sim 9.7 \mu \mathrm{m}$ broad feature is proportional to $A_{V}$ (e.g., Whittet 2003). Although it is not clear that the same correlation holds in the dense protostellar medium (Chiar et al. 2007), we computed a lower limit to the extinction of $A_{V} \gtrsim 30$ by fitting the $\sim 9.7 \mu \mathrm{m}$ absorption band and assuming a single slab geometry.

\subsection{Dust continuum emission}

The dust continuum emission peaks in the far-IR domain at $\sim 100 \mu \mathrm{m}$. This is consistent with the presence of a massive dusty envelope with relatively warm dust temperatures. Using a simple modified blackbody with a dust opacity varying as $\tau_{\lambda}=$ $\tau_{100}(100 / \lambda)^{\beta}$, where $\tau_{100}$ is the continuum opacity at $100 \mu \mathrm{m}$ and $\beta$ is the dust spectral index, we obtain a size of $\sim 7^{\prime \prime}$ for the optically thick far-IR continuum source, with $\tau_{100} \simeq 2.5$, $\beta=1.7, L_{\mathrm{far}-\mathrm{IR}} \simeq 26 L_{\odot}$ and a dust temperature of $T_{\mathrm{d}} \simeq 33 \mathrm{~K}$ (Fig. 2). These parameters agree with more sophisticated radiative transfer models of the continuum emission detected with ISO/LWS, for which a far-IR source size of $\sim 4^{\prime \prime}$ ( $\sim 500 \mathrm{AU}$ in radius) and $T_{\mathrm{d}}=43 \mathrm{~K}$ at the $\tau_{100}=1$ equivalent radius were inferred (Larsson et al. 2000). They are also consistent with more recent SED models (Kristensen et al. 2012) and with the compact $\left(\sim 5^{\prime \prime}\right)$ emission revealed by $\mathrm{mm}$ interferometric observations and thought to arise from the densest regions of the inner envelope and from outflow material (Hogerheijde et al. 1999; Enoch et al. 2009). Note that owing to the large continuum opacity below $100 \mu \mathrm{m}$, observations at these wavelengths probably do not trace the innermost regions, i.e., the developing circumstellar disk with an approximate radius of $\sim 100$ AU (e.g., Rodríguez et al. 2005).

\section{Analysis}

\section{1. ${ }^{12} \mathrm{CO},{ }^{13} \mathrm{CO}, \mathrm{H}_{2} \mathrm{O}$ and $\mathrm{OH}$ Rotational Ladders}

Figure 6 shows all ${ }^{12} \mathrm{CO}$ and ${ }^{13} \mathrm{CO}$ detected lines in a rotational diagram. In this plot we assumed that the line emission arises from a source with a radius of $500 \mathrm{AU}$ (see Sect. 3.3). Given the high densities in the inner envelope of protostars $\left(>10^{6} \mathrm{~cm}^{-3}\right.$; see below), the rotational temperatures $\left(T_{\text {rot }}\right)$ derived from these plots are a good lower limit to $T_{\mathrm{k}}$. The ${ }^{12} \mathrm{CO}$ diagram suggests the presence of 3 different $T_{\text {rot }}$ components with $T_{\text {rot }}=$ $622 \pm 30 \mathrm{~K}\left(\right.$ for $\left.J_{\mathrm{u}} \lesssim 42\right), T_{\text {rot }}=337 \pm 40 \mathrm{~K}\left(J_{\mathrm{u}} \lesssim 26\right)$ and $T_{\text {rot }}=103 \pm 15 \mathrm{~K}\left(J_{\mathrm{u}} \lesssim 14\right)$ respectively. The estimated error in $T_{\text {rot }}$ corresponds to different choices of $J_{\mathrm{u}}$ cutoffs for the different components ${ }^{3}$. In the following we shall refer to them as the hot, warm and cool components. Whether or not these $T_{\text {rot }}$ components are associated with 3 real physical components or with a more continuous temperature and mass distribution will be discussed in Sect. 4.1. Here we note that the submm low$J$ CO emission shows broad line-profiles and a more extended distribution (Davis et al. 1999; Dionatos et al. 2010) than the high- $J \mathrm{CO}$ and $\mathrm{H}_{2} \mathrm{O}$ lines detected with PACS (more sharply peaked near the protostar). In addition, velocity-resolved observations with HIFI of high- $J$ CO lines up to $J=16-15$ show different profiles compared with low- $J$ CO lines (Y1ldiz et al. 2012; Kristensen et al., in prep.). Specifically, for the ${ }^{12} \mathrm{CO} J=$ 10-9 profile toward SMM1, Yildiz et al. (in prep.) find that about $1 / 3$ of the integrated intensity is due to a narrow (FWHM

\footnotetext{
3 Note that an extinction correction of $A_{V}=150$ will only imply slightly larger far-IR line fluxes (more than a factor 1.5 below $\sim 80 \mu \mathrm{m}$ ). Slightly higher rotational temperatures would be inferred if $A_{V}=150$ ( $\sim 350 \mathrm{~K}$ and $\sim 700 \mathrm{~K}$ for the warm and hot components respectively).
} 


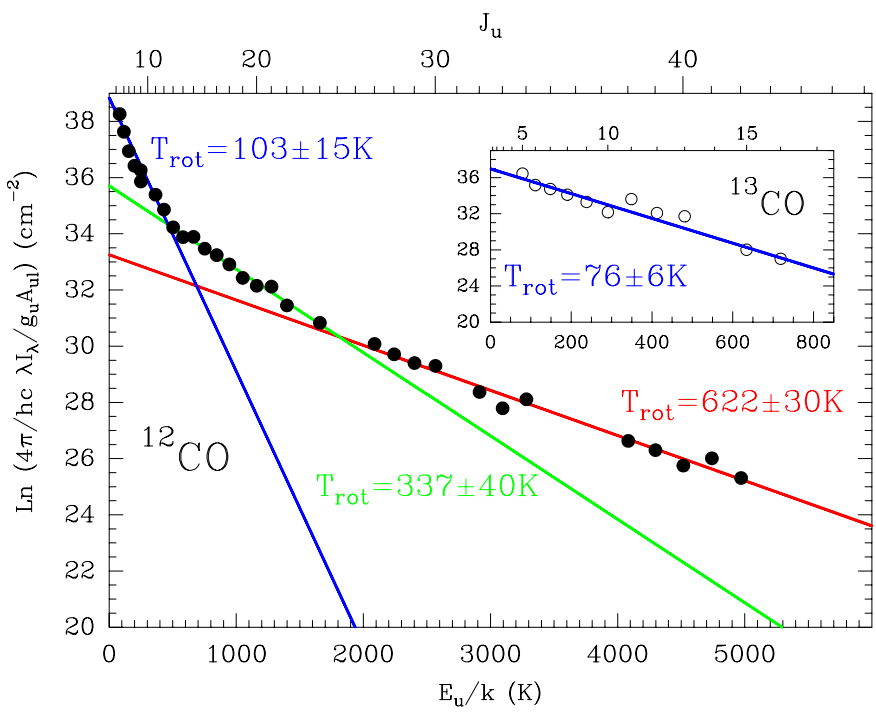

Fig. 6. ${ }^{12} \mathrm{CO}$ and ${ }^{13} \mathrm{CO}$ rotational diagrams obtained from PACS and SPIRE spectra. The estimated error in $T_{\text {rot }}$ for ${ }^{12} \mathrm{CO}$ corresponds to different choices of $J_{\mathrm{u}}$ cutoffs for the different components. The range of possible $T_{\text {rot }}$ for ${ }^{13} \mathrm{CO}$ reflects the standard error on the fitted values.

of a few $\mathrm{km} \mathrm{s}^{-1}$ ) component originating from the quiescent envelope and $2 / 3$ to the broad outflow component. Thus, the cool gas component seen in the SPIRE submm maps is dominated by the entrained outflow gas. On the other hand, the CO $J=16-15$ profile of SMM1 is less broad and more similar to the excited $\mathrm{H}_{2} \mathrm{O}$ line profiles observed with HIFI (Kristensen et al., in prep.). Thus, the far-IR $\mathrm{CO}$ and $\mathrm{H}_{2} \mathrm{O}$ lines detected by PACS clearly probe different physical components than the SPIRE data.

Alternatively, Neufeld (2012) pointed out that the shape of the rotational diagrams do not, by themselves, necessarily require multiple temperature components. In particular, a single$T_{\text {rot }}$ solution could be found for the ${ }^{12} \mathrm{CO} J_{\mathrm{u}}>14$ lines in Serpens SMM1 if the gas were very hot $\left(T_{\text {kin }} \simeq 2500 \mathrm{~K}\right)$ but had a low density (a few $10^{4} \mathrm{~cm}^{-3}$ ). This scenario seems less likely, at least for the circumstellar gas in the vicinity of the protostar. Note that the gas density in the inner envelope of SMM1 is necessarily higher, as probed by our detection of high- $J$ lines from high dipole moment molecules such as $\mathrm{HCN}$ and $\mathrm{HCO}^{+}$(with very high critical densities). In addition, detailed SED models predict densities of $n\left(\mathrm{H}_{2}\right) \simeq 4 \times 10^{6} \mathrm{~cm}^{-3}$ at $\sim 1000 \mathrm{AU}$, whereas densities of the order of $\sim 10^{5} \mathrm{~cm}^{-3}$ are only expected at much larger radii (Kristensen et al. 2012). Therefore, it seems more likely that the different $T_{\text {rot }}$ slopes probe different physical components or the presence of a temperature gradient.

A rotational diagram of the detected ${ }^{13} \mathrm{CO}$ lines provides $T_{\text {rot }}=76 \pm 6 \mathrm{~K}$, thus lower than the $T_{\text {rot }}$ inferred for ${ }^{12} \mathrm{CO}$ in the cool component. The measured ${ }^{12} \mathrm{CO} /{ }^{13} \mathrm{CO} J=5-4$ line intensity ratio is $\simeq 8$, thus much lower than the typical ${ }^{12} \mathrm{C} /{ }^{13} \mathrm{C}$ isotopic ratio of $\sim 60$ Langer \& Penzias (1990), whereas the ${ }^{12} \mathrm{CO} /{ }^{13} \mathrm{CO} J=16-15$ intensity ratio is $\simeq 55$. These different ratios show that the submm ${ }^{12} \mathrm{CO}$ low- and mid- $J$ lines are optically thick, but the high- $J$ far-IR lines are optically thin.

An equivalent plot of the observed far-IR $\mathrm{H}_{2} \mathrm{O}$ line intensities in a rotational diagram gives $T_{\text {rot }}=136 \pm 27 \mathrm{~K}$ without much indication of multiple $T_{\text {rot }}$ components (Fig. 7). Since $\mathrm{H}_{2} \mathrm{O}$ critical densities are much higher than those of $\mathrm{CO}$, collisional thermalization only occurs at very high densities $\left(>10^{8} \mathrm{~cm}^{-3}\right)$. Therefore, the large scatter of the $\mathrm{H}_{2} \mathrm{O}$ rotational diagram is a consequence of subthermal excitation conditions

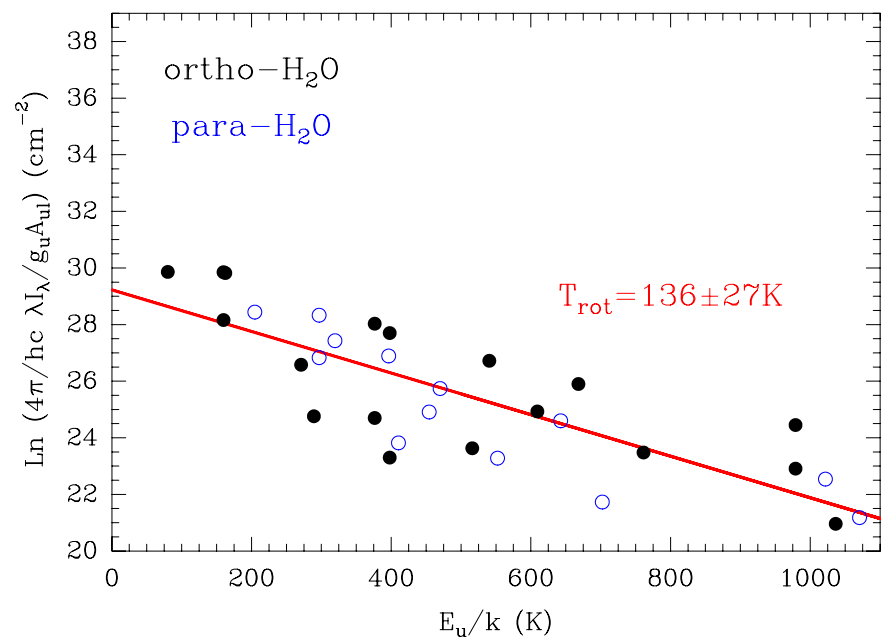

Fig. 7. $\mathrm{H}_{2} \mathrm{O}$ rotational diagram showing $o-\mathrm{H}_{2} \mathrm{O}$ (filled circles) and $p-\mathrm{H}_{2} \mathrm{O}$ far-IR lines (open circles). Owing to subthermal excitation $\left(T_{\text {rot }} \ll T_{\mathrm{k}}\right)$ and large opacities of most lines, the $\mathrm{H}_{2} \mathrm{O}$ diagram displays a larger scatter (standard error on the fitted values are shown) than $\mathrm{CO}$.

( $T_{\text {rot }} \ll T_{\mathrm{k}}$ ) and large $\mathrm{H}_{2} \mathrm{O}$ line opacities. We anticipate here that the opacity of most (but not all) far-IR $\mathrm{H}_{2} \mathrm{O}$ lines is very high $(\tau \gg 1)$. Compared to $\mathrm{CO}$, the $\mathrm{H}_{2} \mathrm{O}$ rotational diagram is thus obviously less meaningful. A similar rotational diagram of the observed $\mathrm{OH}$ lines provides $T_{\text {rot }}=72 \pm 8 \mathrm{~K}$ (see also Wampfler et al. 2012), even lower than the inferred $\mathrm{H}_{2} \mathrm{O}$ rotational temperature. Like $\mathrm{H}_{2} \mathrm{O}, \mathrm{OH}$ transitions also have high critical densities and line opacities (except the high-energy and the cross-ladder transitions).

\subsection{Simple model of the far-IR and submm line emission}

Observations and models of the protostellar environment suggest that the observed emission can arise from different physical components, where different mechanisms dominate the gas heating and the prevailing chemistry: hot and warm gas from energetic shocks in the inner envelope (e.g., small scale shocks along the outflow cavity walls, bow shocks and working surfaces within an atomic jet); UV-illuminated gas in the cavity walls; cooler gas from the envelope passively heated by the protostar luminosity and the entrained outflow gas (e.g., van Dishoeck et al. 2011). Unfortunately, the lack of enough spectral and angular resolution of our data does not allow us to provide a complete characterization of all the different possible components. However, the large number of detected lines, high excitation $\left(E_{\mathrm{u}} / k>300 \mathrm{~K}\right)$ optically thin lines in particular, helps to determine the dominant contributions and the average physical conditions.

With this purpose, we have carried out a non-LTE radiative transfer model of the central spaxel far-IR and submm spectrum (the protostar position) using a multi-molecule LVG code (Cernicharo 2012). In this model we included three spherical components suggested by the three temperature components seen in the ${ }^{12} \mathrm{CO}$ rotational diagram (i.e., hot, warm and cool components). In our model, $\mathrm{CO}$ is present in the three components, but since a single rotational temperature roughly explains the observed far-IR $\mathrm{H}_{2} \mathrm{O}$ and $\mathrm{OH}$ lines, most of their emission in the PACS domain can be reproduced with only one component, the hot component for $\mathrm{H}_{2} \mathrm{O}$ and the warm component for $\mathrm{OH}$ (see next section for more details). Note that we do not model the extended quiescent envelope with the bulk of the (cold) mass seen in the $\mathrm{mm}$ continuum and narrow $\mathrm{C}^{18} \mathrm{O}$ line 
emission (Y1ldiz et al., in prep.). Foreground absorption and emission from the low density and low temperature extended envelope are thus not included in the model but they will basically influence narrow velocity ranges of the lowest energy-level lines.

The latest available collisional rates were used (e.g., Daniel et al. 2011, and references therein for $\mathrm{H}_{2} \mathrm{O}$; and Yang et al. 2010; extended by Neufeld 2012, for CO). Although only one line of $o-\mathrm{H}_{2}$ and one line of $p-\mathrm{H}_{2}$ are detected towards the Serpens SMM1 protostar position, the large $\mathrm{H}_{2} \quad 0-0 \mathrm{~S}(2) / \mathrm{S}(1)$ line intensity ratio suggests a $\mathrm{H}_{2}$ ortho-topara (OTP) ratio lower than 3 in the gas probed by these low excitation $\mathrm{H}_{2}$ lines. Here we shall adopt an OTP ratio for $\mathrm{H}_{2}$ of 1 (the value we obtain from the two observed $\mathrm{H}_{2}$ lines assuming LTE and a rotational temperature of $\sim 800 \mathrm{~K}$ ). Note that such low non-equilibrium $\mathrm{H}_{2}$ OTP values have been inferred, for example, in the hot shocked gas towards HH54 and HH7-11 (Neufeld et al. 2006). The water vapour OTP ratio in the model, however, is let as a free parameter.

The $o-\mathrm{H}_{2} \mathrm{O}$ ground-state line observed with HIFI towards Serpens SMM1 shows a two component emission line profile with a medium component $\left(\Delta v \simeq 15 \mathrm{~km} \mathrm{~s}^{-1}\right)$ thought to arise from small-scale shocks in the inner cavity walls and a broad component $\left(\Delta v \simeq 40 \mathrm{~km} \mathrm{~s}^{-1}\right)$ from more extended shockedgas along the outflow (Kristensen et al. 2012). In addition, velocity-resolved observations of the $\mathrm{OH}^{2} \Pi_{1 / 2} J=3 / 2-1 / 2$ line $(\sim 163.1 \mu \mathrm{m})$ with HIFI show a broad line-profile with $\Delta v \simeq$ $20 \mathrm{~km} \mathrm{~s}^{-1}$ (Wampfler 2012, priv. comm.). For simplicity here we shall adopt a typical line-width of $20 \mathrm{~km} \mathrm{~s}^{-1}$ in all modelled components. As in any LVG calculation, the model is more accurate for (effectively) optically thin lines (high- $J$ CO lines, excited and weak $\mathrm{H}_{2} \mathrm{O}$ and $\mathrm{OH}$ lines, etc.) than for very opaque lines (e.g., low excitation lines).

\subsubsection{Model: hot and warm component}

Following Sect. 3.3. and previous far-IR studies of this source, we have assumed a size of $\sim 4^{\prime \prime}(\sim 500$ AU in radius; e.g., Larsson et al. 2000; Kristensen et al. 2012) for the far-IR source (hot and warm components), a mixture of shocked and UV-illuminated gas (see Sect. 5). A good fit to the high- $J{ }^{12} \mathrm{CO}$ emission is obtained for $n\left(\mathrm{H}_{2}\right) \approx 5 \times 10^{6} \mathrm{~cm}^{-3}$ with $T_{\mathrm{k}} \simeq 800 \mathrm{~K}$ and $\simeq 375 \mathrm{~K}$ for the hot and warm components respectively. The approximate mass in these components is only $M \lesssim 0.03 M_{\odot}$. Although this solution is not unique, this model satisfactorily reproduces not only the ${ }^{12} \mathrm{CO}$ high- $J$ lines but also the lines from other species (note that we searched for a combination of $T_{\mathrm{k}}$ and $n\left(\mathrm{H}_{2}\right)$ that reproduces all species simultaneously; see below).

In order to constrain the density and place $\mathrm{H}_{2} \mathrm{O}$ and $\mathrm{OH}$ in a particular model component, we first run a grid of excitation models and investigated how particular line ratios change with $n\left(\mathrm{H}_{2}\right)$ and $T_{\mathrm{k}}$. In this comparison we choose faint, lowopacity lines arising from high energy levels that are observed with a similar PSF. Figure A. 1 shows the variation of the $o-\mathrm{H}_{2} \mathrm{O}$ $3_{30}-3_{03} / 6_{15}-3_{05}(67.269 / 82.031 \mu \mathrm{m})$ and $p-\mathrm{H}_{2} \mathrm{O} 6_{06}-5_{15} / 5_{15}-4_{04}$ $(83.284 / 95.627 \mu \mathrm{m})$ line ratios. The former ratio shows that the excited $\mathrm{H}_{2} \mathrm{O}$ lines arise from dense gas, whereas the latter requires high temperatures $(>500 \mathrm{~K})$. The intersection of the two line ratios is consistent with densities higher than a few $10^{6} \mathrm{~cm}^{-3}$ and temperatures around $\sim 800 \mathrm{~K}$. Note that for $T_{\mathrm{k}}<400 \mathrm{~K}$, the predicted emission from many excited $\mathrm{H}_{2} \mathrm{O}$ lines below $\sim 100 \mu \mathrm{m}$ would be too faint. On the other hand, Fig. A.2 shows a very clear dependence of the $\mathrm{OH} 65.279 / 96.363 \mu \mathrm{m}$ line ratio with the gas temperature. The observed ratio does suggest that $\mathrm{OH}$ arises from gas at $T_{\mathrm{k}} \lesssim 400 \mathrm{~K}$. Following the previous excitation analysis, and according to the higher observed rotational temperatures $\left(T_{\text {rot }}^{\mathrm{H}_{2} \mathrm{O}}>T_{\text {rot }}^{\mathrm{OH}}\right)$ and higher $\mathrm{H}_{2} \mathrm{O}$ energy levels detected, we placed $\mathrm{H}_{2} \mathrm{O}$ in the hot component and $\mathrm{OH}$ (together with $[\mathrm{OI}]$ ) in the warm component. Although this choice provides a better fit to the data, we can not obviously conclude that the bulk of the water vapour and $\mathrm{OH}$ emission arise from different physical components.

Far-IR radiative pumping is not included in our models and thus the derived $\mathrm{H}_{2} \mathrm{O}$ and $\mathrm{OH}$ column densities might be considered as upper limits. Nevertheless, all $\mathrm{H}_{2} \mathrm{O}$ and $\mathrm{OH}$ lines are observed in emission and show $T_{\text {rot }}>T_{\mathrm{d}}$ (where $T_{\mathrm{d}}$ is the dust temperature inferred from the SED analysis, see Sect. 3.3), suggesting that owing to the high densities but moderate far-IR radiation field at $\gtrsim 500 \mathrm{AU}$, collisions dominate at first order. Note that high-mass protostars produce much stronger far-IR dust continuum fields (by orders of magnitude) and many far-IR $\mathrm{OH}$ and water vapour lines are often observed in absorption or show P-Cygni profiles when far-IR pumping dominates the excitation (see e.g., Goicoechea et al. 2006; Cernicharo et al. 2006, for far-IR $\mathrm{OH}$ and $\mathrm{H}_{2} \mathrm{O}$ lines in Orion $\mathrm{KL}$ outflows).

\subsubsection{Model: cool gas component}

In order to fit the more extended ${ }^{12} \mathrm{CO}$ low- and mid- $J$ emission and to match the $T_{\text {rot }} \simeq 100 \mathrm{~K}$ component seen in the ${ }^{12} \mathrm{CO}$ rotational diagram (Fig. 6), we included a third cool component the entrained outflow gas. From the SPIRE ${ }^{12} \mathrm{CO}$ maps we infer an approximate radius of $\sim 20^{\prime \prime}(\sim 4500 \mathrm{AU})$ for the emitting region. For this geometry, a density of $n\left(\mathrm{H}_{2}\right) \approx 2 \times 10^{5} \mathrm{~cm}^{-3}$ and a temperature of $T_{\mathrm{k}} \simeq 140 \mathrm{~K}$ produces a good fit of the observed ${ }^{12} \mathrm{CO}$ submm emission (see also Yildiz et al. 2012, for velocityresolved observations in NGC 1333 IRAS 4A/4B). This component is also needed to fit the lower excitation $\mathrm{H}_{2} \mathrm{O}$ submm lines.

Low- and mid- $J{ }^{13} \mathrm{CO}$ lines are optically thin and thus, in addition to the swept-up or entrained outflow gas, velocityresolved submm ${ }^{13} \mathrm{CO}$ line profiles would carry information about other components such as the UV-heated gas and the quiescent envelope, which may well dominate the ${ }^{13} \mathrm{CO}$ lower$J$ emission (Y1ldiz et al. 2012). Detailed models of the emission from the extended and passively heated envelope (having most of the mass) predict ${ }^{12} \mathrm{CO}$ rotational temperatures around $\simeq 30-60 \mathrm{~K}$ (Visser et al. 2012; Harsono et al., in prep.).

To summarize, Table 2 shows the model parameters and Fig. 8 shows the entire $\sim 55$ to $671 \mu \mathrm{m}$ synthetic spectrum for the hot (red), warm (green) and cool (blue) components convolved with the PACS and SPIRE resolutions. A comparison of the resultant synthetic spectrum (by simply adding the 3 component spectra) with the Herschel spectra is shown in Fig. 9.

\subsubsection{Columns, abundances and validity of the model}

Owing to the lack of angular resolution to determine the beam filling factors of the different physical components towards Serpens SMM1, the relative abundance ratios derived from the model and shown in Table 3 are a better diagnostic tool than the absolute column densities. Nevertheless, here we provide the source-averaged column densities $(N)$ in the different components as well as the upper limits for several non-detected species (Table 2). In the hot component we find $\sim 5 \times 10^{16} \mathrm{~cm}^{-2}$ and $\sim 2 \times 10^{16} \mathrm{~cm}^{-2}$ column densities for ${ }^{12} \mathrm{CO}$ and $\mathrm{H}_{2} \mathrm{O}$ respectively. Note that a water vapour OTP ratio of $\sim 3$ provides the best fit to the observed water vapour lines and this is the value adopted in the models. Assuming that the gas 
J. R. Goicoechea et al.: The complete spectrum of the Class 0 protostar Serpens SMM1

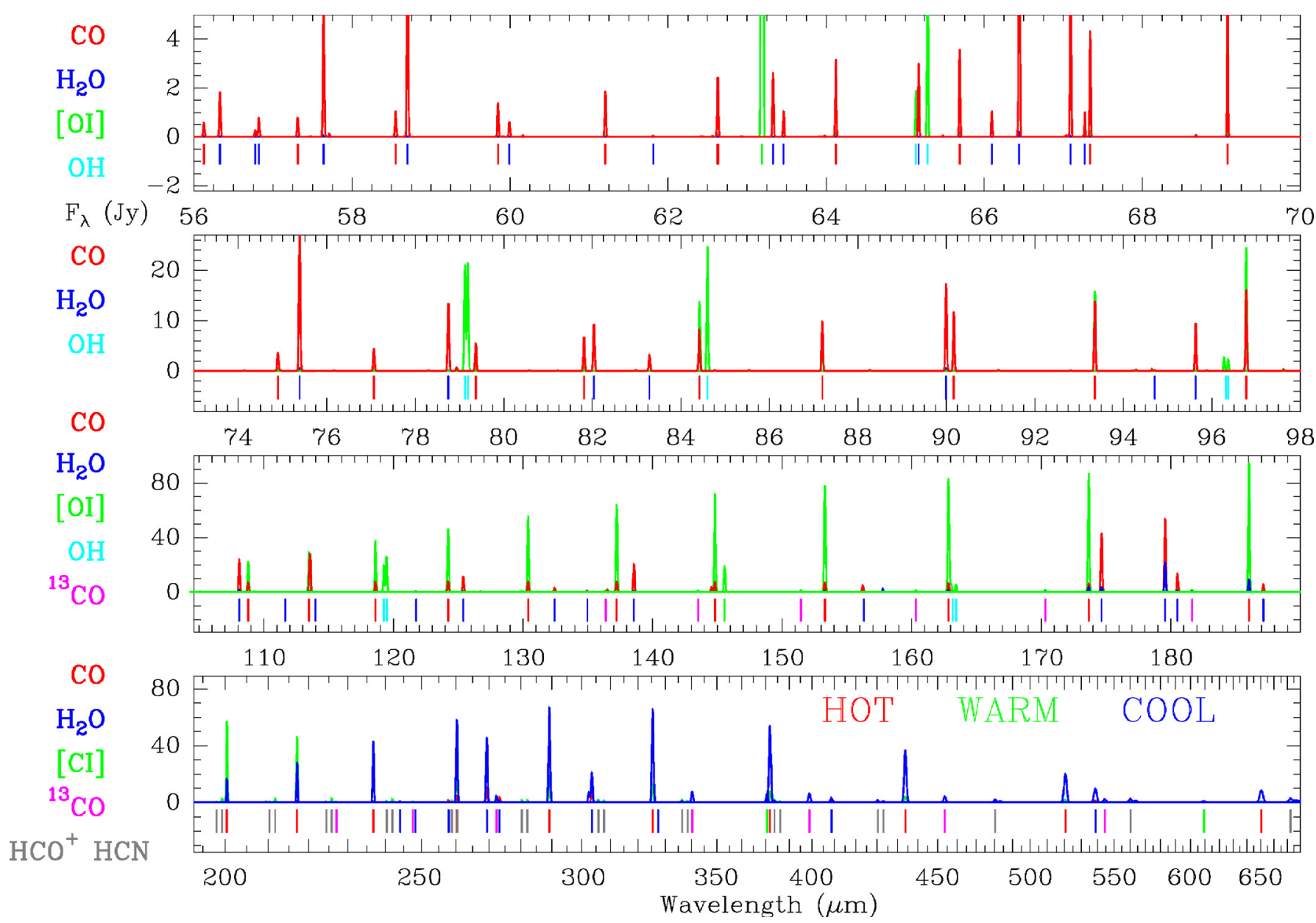

Fig. 8. Synthetic non-LTE LVG spectrum of the Class 0 protostar Serpens MM1 convolved to PACS (top three panels) and SPIRE (lower panel) spectral resolutions. Continuous curves show the line emission contribution from the "hot" (red), "warm" (green) and "cool" (blue) components discussed in the text. Vertical labels mark the wavelength position of $\mathrm{CO}$ (red), $\mathrm{H}_{2} \mathrm{O}$ (blue), $\mathrm{OH}$ (cyan), ${ }^{13} \mathrm{CO}$ (magenta), $\mathrm{HCO}^{+}$and $\mathrm{HCN}$ (grey) rotational transitions. [O I]63, 145 and [C I]370, $609 \mu \mathrm{m}$ fine structure lines are marked with green labels.

Table 2. Model components and source-averaged column densities.

\begin{tabular}{lccc}
\hline \hline Comp. & Radius & $T_{\mathrm{k}}(\mathrm{K})$ & $n\left(\mathrm{H}_{2}\right)\left(\mathrm{cm}^{-3}\right)$ \\
\hline Hot & $\sim 500 \mathrm{AU}$ & 800 & $5 \times 10^{6}$ \\
\hline$N\left(\mathrm{~cm}^{-2}\right)$ & ${ }^{12} \mathrm{CO}\left(5 \times 10^{16}\right)$ & $\mathrm{H}_{2} \mathrm{O}\left(2 \times 10^{16}\right)$ & \\
& ${ }^{13} \mathrm{CO}\left(9 \times 10^{14}\right)$ & & \\
\hline Warm & $\sim 500 \mathrm{AU}$ & 375 & $5 \times 10^{6}$ \\
\hline$N\left(\mathrm{~cm}^{-2}\right)$ & ${ }^{12} \mathrm{CO}\left(1 \times 10^{18}\right)$ & ${ }^{13} \mathrm{CO}\left(2 \times 10^{16}\right)$ & \\
& $\mathrm{OH}\left(1 \times 10^{16}\right)$ & $\mathrm{HCO}^{+}\left(5 \times 10^{14}\right)$ & \\
& $\mathrm{HCN}\left(2 \times 10^{14}\right)$ & $\mathrm{CH}^{+}\left(<2 \times 10^{14}\right)$ & \\
& $\mathrm{CO}^{+}\left(<2 \times 10^{15}\right)$ & $\mathrm{NH}_{3}\left(<2 \times 10^{15}\right)$ & \\
& $\mathrm{O}\left(1 \times 10^{18}\right)$ & & $2 \times 10^{5}$ \\
\hline Cool & $\left.\sim 4500 \mathrm{AU}^{-2}\right)$ & 140 & \\
\hline$N\left(\mathrm{~cm}^{-2}\right)$ & ${ }^{12} \mathrm{CO}\left(1 \times 10^{17}\right)$ & $\mathrm{H}_{2} \mathrm{O}\left(8 \times 10^{15}\right)$ & \\
& ${ }^{13} \mathrm{CO}\left(1 \times 10^{16}\right)$ & $\mathrm{HCO}^{+}\left(2 \times 10^{14}\right)$ & \\
& $\mathrm{HCN}\left(1 \times 10^{15}\right)$ & $\mathrm{C}\left(1 \times 10^{17}\right)$ & \\
& $\mathrm{C}^{+}\left(1 \times 10^{16}\right)$ & & \\
\hline
\end{tabular}

Notes. We assume a line-width of $20 \mathrm{~km} \mathrm{~s}^{-1}$ in all components.

in the warm component $\left(T_{\mathrm{k}} \simeq 375 \mathrm{~K}\right)$ covers a similar area, we obtain $N(\mathrm{OH}) \sim 10^{16} \mathrm{~cm}^{-2}$ and $N\left({ }^{12} \mathrm{CO}\right) \sim 10^{18} \mathrm{~cm}^{-2}$ (a factor $\approx 20$ larger than $N\left({ }^{12} \mathrm{CO}\right)$ in the hot component).

The detected mid-IR $\mathrm{H}_{2}$ lines provide a lower limit to the $\mathrm{H}_{2}$ column density of $N_{\mathrm{H}_{2}} \approx 10^{22} \mathrm{~cm}^{-2}$. We use this column density to provide an upper limit to the absolute abundances (with respect to $\mathrm{H}_{2}$ ) in the hot+warm components. In particular we obtain $\lesssim 10^{-4}, \lesssim 0.2 \times 10^{-5}$ and $\lesssim 10^{-6}$ for the $\mathrm{CO}, \mathrm{H}_{2} \mathrm{O}$ and $\mathrm{OH}$ abundances respectively. The inferred upper limit to the water vapour abundance is much higher than the $\approx 10^{-(8-9)}$ value typically found in cold interstellar clouds (e.g., Caselli et al. 2010 ) but is lower than the $\approx 10^{-4}$ value often expected in the hot shocked gas (e.g., Kaufman \& Neufeld 1996). Note that the high source-averaged column density of atomic oxygen $\left(\sim 10^{18} \mathrm{~cm}^{-2}\right)$ suggests that a significant fraction of gas-phase oxygen reservoir in shocks is kept in atomic form.

The column densities in the entrained outflow gas (cool component at $T_{\mathrm{k}} \simeq 140 \mathrm{~K}$ ) are given in Table 2 . The absolute columns are more uncertain as they depend on the assumed spatial distribution of the swept-up outflow gas. Note that in order to fit the low- $J{ }^{13} \mathrm{CO}$ lines in the cool component, we had to include a low ${ }^{12} \mathrm{CO} /{ }^{13} \mathrm{CO}$ column density ratio of $\simeq 10$, confirming that the low- $J{ }^{12} \mathrm{CO}$ lines are optically thick and thus they do not probe the bulk of the material seen in the low- $J$ CO isotopologue line emission (i.e., the massive and quiescent envelope).

Our simple LVG model satisfactorily reproduces the absolute fluxes of most observed lines and does not predict lines that are not detected in the spectra (see Fig. 9). Despite the fact that this model solution is obviously not unique, the agreement with observations suggests that this model captures the average physical conditions of the shocked gas near the protostar. The level 


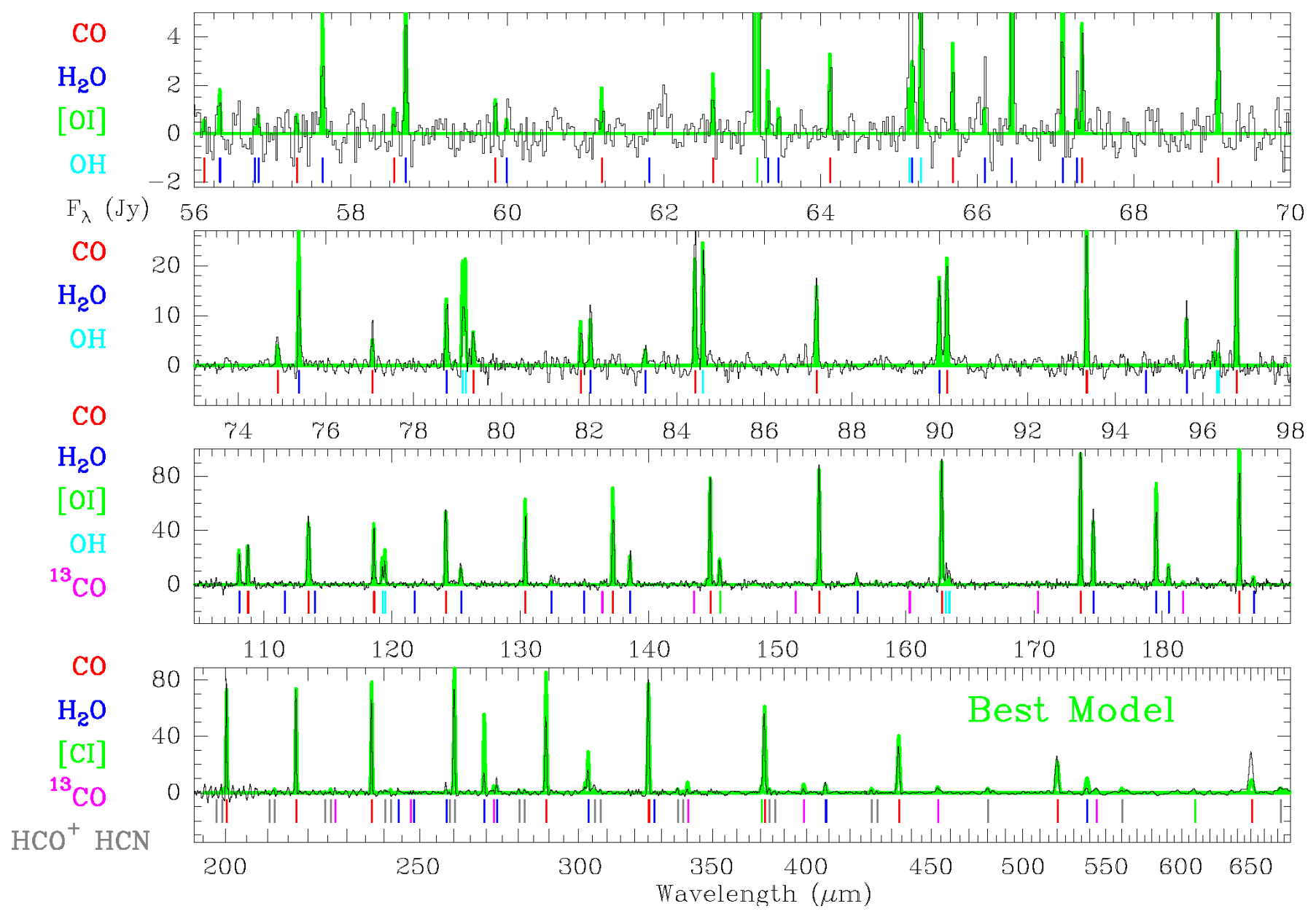

Fig. 9. Herschel far-IR and submm spectrum of Serpens SMM1 at the central spaxel position (black histogram) and complete model (green continuous curves) adding the emission from the hot, warm and cool components and convolved to PACS and SPIRE spectral resolutions.

Table 3. Selected abundance ratios in the modelled components.

\begin{tabular}{lcc}
\hline \hline \multicolumn{1}{c}{ Species } & Abundance ratio & Component \\
\hline $\mathrm{H}_{2} \mathrm{O}_{\mathrm{h}} /\left(\mathrm{CO}_{\mathrm{w}}+\mathrm{CO}_{\mathrm{h}}\right)$ & $\sim 0.02$ & hot and warm \\
$\mathrm{OH}_{\mathrm{w}} /\left(\mathrm{CO}_{\mathrm{w}}+\mathrm{CO}_{\mathrm{h}}\right)$ & $\sim 0.01$ & hot and warm \\
$\mathrm{O}_{\mathrm{w}} /\left(\mathrm{CO}_{\mathrm{w}}+\mathrm{CO}_{\mathrm{h}}\right)$ & $\sim 0.8$ & hot and warm \\
$\mathrm{OH}_{\mathrm{w}} / \mathrm{H}_{2} \mathrm{O}_{\mathrm{h}}$ & $\leq 0.5$ & warm $/$ hot \\
$\mathrm{CO}_{\mathrm{w}} / \mathrm{CO}_{\mathrm{h}}$ & $\sim 20$ & warm $/$ hot \\
$\mathrm{H}_{2} \mathrm{O}_{\mathrm{h}} / \mathrm{CO}_{\mathrm{h}}$ & $\sim 0.4$ & hot \\
$\mathrm{CH}_{\mathrm{h}}^{+} / \mathrm{H}_{2} \mathrm{O}_{\mathrm{h}}$ & $<0.01$ & hot \\
$\mathrm{O}_{\mathrm{w}} / \mathrm{OH}_{\mathrm{w}}$ & $\sim 100$ & warm \\
$\mathrm{HCO}_{\mathrm{w}}^{+} / \mathrm{HCN}_{\mathrm{w}}$ & $\sim 2.5$ & warm \\
$\mathrm{HCO}_{\mathrm{c}}^{+} / \mathrm{HCN}_{\mathrm{c}}$ & $\sim 0.2$ & cool \\
$\mathrm{H}_{2} \mathrm{O}_{\mathrm{c}} / \mathrm{CO}_{\mathrm{c}}$ & $<0.08$ & cool \\
\hline
\end{tabular}

of agreement is typically better than $\sim 30 \%$ (i.e., similar to the calibration uncertainty). The worst agreement occurs for several low-excitation optically thick lines that can arise from different physical components because the non-local radiative coupling between different components is not treated in the LVG model. In addition, some lines such as the $\sim 163 \mu \mathrm{m} \mathrm{OH}$ lines (sensitive to far-IR pumping, see Offer \& van Dishoeck 1992; Goicoechea \& Cernicharo 2002) are underestimated by $\gtrsim 40 \%$, suggesting that radiative pumping may play some role (see detailed OH models by Wampfler et al. 2012).

Regarding the $T_{\mathrm{k}}$ and $n\left(\mathrm{H}_{2}\right)$ conditions inferred in each component, Fig. A.3 in the Appendix shows that for a given gas density, we can distinguish temperature variations of $\sim 30 \%$ (especially in high- $J \mathrm{CO}$ and in some high excitation $\mathrm{H}_{2} \mathrm{O}$ and $\mathrm{OH}$ lines). Figure A.4 shows that for a given gas temperature, density variations of a factor $\sim 2-3$ can also be distinguished. Therefore, for the assumed geometry and physical conditions, the derived source-averaged column densities are accurate within a factor of $\sim 2$.

\section{Discussion}

\subsection{Shocked-gas components}

Several fine-structure lines that probe the very hot atomic gas (a few thousand $\mathrm{K}$ ) near the protostar are detected in the midIR. Besides, most tracers of the shock-heated molecular gas (a few hundred K) appear in the far-IR. At longer submm and $\mathrm{mm}$ wavelengths, extended emission from cool entrained outflow gas and from the cold massive envelope dominates. The relative intensities of the detected mid- and far-IR atomic and molecular lines help to qualitatively constrain the nature of the main shocked-gas components in Serpens SMM1.

The shock wave velocity, pre-shock density and the magnetic field strength determine most of the shocked gas properties. Fast shocks can destroy molecules and ionize atoms, whereas slower shocks heat the gas without destroying molecules. Depending on the evolution of the shock structure it is common to distinguish between $J$-type (or Jump) and $C$-type (or Continuous). More complicated, "mixed" non-stationary situations may also exist 
(see reviews by e.g., Draine \& McKee 1993; Walmsley et al. 2005). As we show below, our observations suggest the presence of both fast and slow shocks in Serpens SMM1.

\subsubsection{Fast shocks}

The bright and velocity-shifted [O I]63 $\mu \mathrm{m}$ emission, together with the detection of $[\mathrm{Ne} \mathrm{II}] 12 \mu \mathrm{m}$ and very high energy [Fe II] lines in Serpens SMM1 suggests the presence of fast dissociative $J$-shocks related with the presence of an embedded atomic jet near the protostar. Note that [Ne II] and [Fe II] lines have been detected towards other YSOs (see e.g., Lahuis et al. 2010, for the c2d Spitzer sample). Hollenbach \& McKee (1989) presented detailed models for the fine-structure emission of atoms and ions in such dissociative $J$-type shocks. However, the chemistry of $\mathrm{S}, \mathrm{Fe}, \mathrm{Si}$ and related molecules (including gas-phase depletion, e.g., Nisini et al. 2005) were not included and thus the fine-structure absolute line intensity predictions are likely upper limits. In addition, because the beam filling factors of the possible shock components are not known, line ratios provide a much better diagnostic than absolute intensities. According to these detailed models, the observed [Si II]35 $\mu \mathrm{m} /[\mathrm{Fe}$ II $] 26 \mu \mathrm{m}<1$ line intensity ratio (independently on the assumed extinction) provides a lower limit to the pre-shock density, $n_{0}=n_{0}(\mathrm{H})+2 n_{0}\left(\mathrm{H}_{2}\right)$, of $>10^{4} \mathrm{~cm}^{-3}$. Besides, owing to the high ionization potential of $\mathrm{Ne}^{+}$, the $[\mathrm{Ne}$ II $] 12 \mu \mathrm{m} /[\mathrm{Fe}$ II $] 26 \mu \mathrm{m}$ line intensity ratio increases sharply with the shock velocity $\left(v_{\mathrm{s}}\right)$. A lower limit of $v_{\mathrm{s}}>60 \mathrm{~km} \mathrm{~s}^{-1}$ is found from the observed $[\mathrm{Ne}$ II $] 12 \mu \mathrm{m} /[\mathrm{Fe}$ II $] 26 \mu \mathrm{m} \geq 0.1$ line ratio (applying an extinction correction of $A_{V} \geq 30$ ).

Models of fast dissociative $J$-shocks predict that the gas is initially atomic and very hot (a few thousand $\mathrm{K}$ ), but by the time that molecules reform, the gas cools to about $400 \mathrm{~K} . \mathrm{H}_{2}$ formation provides the main heat source for this "temperature plateau" (Hollenbach \& McKee 1989; Neufeld \& Dalgarno 1989). In such models, $\mathrm{H}_{2}$ is not so abundant and cooling by $\mathrm{H}_{2}$ and $\mathrm{H}_{2} \mathrm{O}$ can be less important than by [OI], $\mathrm{CO}$ and $\mathrm{OH}$. Therefore, in addition to the mid-IR fine-structure emission, a significant fraction of the $[\mathrm{OI}], \mathrm{OH}$ and the $\mathrm{CO}$ warm component emission can arise behind fast dissociative shocks triggered by a jet impacting the inner, dense evelope.

\subsection{2. (UV-irradiated) slow shocks}

From the observed $\mathrm{H}_{2} \mathrm{O}-0 \mathrm{~S}(2)$ weak line and the upper limit of the non-detected $\mathrm{S}(0)$ line, we derive a lower limit to the $\mathrm{H}_{2}$ rotational temperature $\left(T_{42}\right)$ of $\sim 700 \mathrm{~K}$ (if a extinction correction of $A_{V}=30$ is applied). This (and higher) temperatures of the molecular gas are often inferred behind non-dissociative shocks (e.g., Neufeld et al. 2006; Melnick et al. 2008).

Depending on the shock velocity, non-dissociative $C$-type shocks shielded from UV radiation can produce very high gas temperatures without destroying molecules $\left(T_{\mathrm{k}} \sim 400-3000 \mathrm{~K}\right.$ for $v_{\mathrm{s}} \sim 10-40 \mathrm{~km} \mathrm{~s}^{-1}$ in the models by Kaufman \& Neufeld 1996). They also naturally produce high $\mathrm{H}_{2} \mathrm{O} / \mathrm{CO}$ abundance ratios (close to 1 ) but predict low $\mathrm{OH} / \mathrm{H}_{2} \mathrm{O} \ll 1$ ratios (owing to negligible $\mathrm{H}_{2} \mathrm{O}$ photodissociation but very efficient $\mathrm{OH}+$ $\mathrm{H}_{2} \rightarrow \mathrm{H}_{2} \mathrm{O}+\mathrm{H}$ reactions in the hot molecular gas). Therefore, a non-dissociative $C$-shock could be the origin of the observed $\mathrm{H}_{2}$ lines, high- $J \mathrm{CO}\left(J_{\mathrm{u}} \gtrsim 30\right)$ and excited $\mathrm{H}_{2} \mathrm{O}$ lines $\left(E_{\mathrm{u}} / k>300 \mathrm{~K}\right)$. Note that in our simple model we infer a high $\mathrm{H}_{2} \mathrm{O}_{\mathrm{h}} / \mathrm{CO}_{\mathrm{h}} \simeq 0.4$ relative abundance when considering

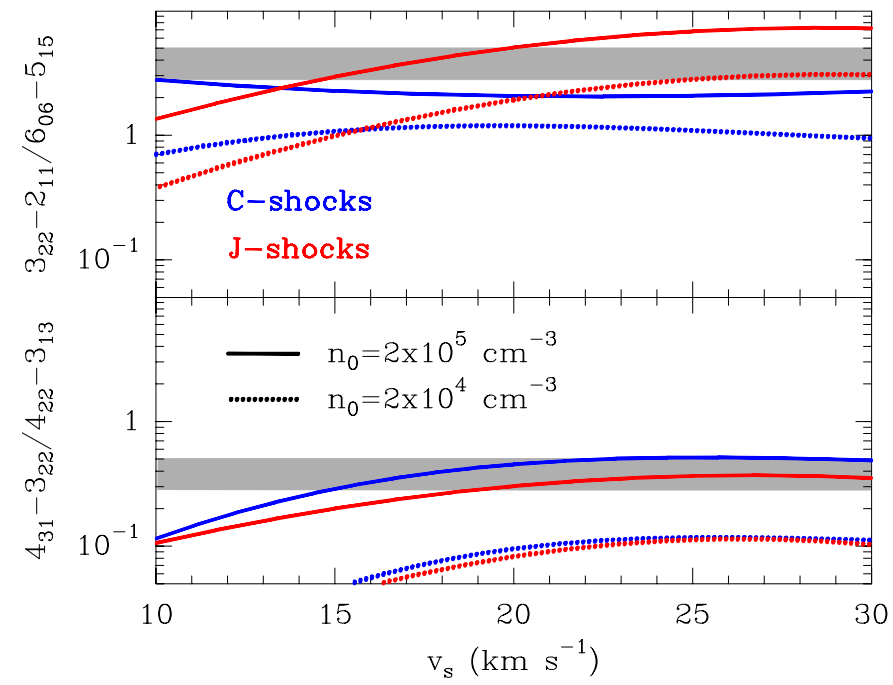

Fig. 10. Comparison of selected $p-\mathrm{H}_{2} \mathrm{O}$ line intensity ratios observed with PACS and shock model predictions from Flower \& Pineau Des Forêts (2010). Red and blue curves represent $J$-type and $C$-type shock models respectively. The pre-shock gas density is $n_{0}=$ $2 \times 10^{5} \mathrm{~cm}^{-3}$ (continuous curves) and $n_{0}=2 \times 10^{4} \mathrm{~cm}^{-3}$ (dotted curves). Low opacity lines from high energy rotational levels $\left(E_{\mathrm{u}} / k \sim\right.$ 300-650 K) observed at similar wavelengths are selected. The upper panel shows the $p-\mathrm{H}_{2} \mathrm{O} 3_{22}-2_{11} / 6_{06}-5_{15}$ line ratio $(89.988 / 83.284 \mu \mathrm{m})$ and the lower panel shows the $4_{31}-3_{22} / 4_{22}-3_{13}(56.325 / 57.637 \mu \mathrm{m})$ line ratios. The grey horizontal areas show the observed ratios and their error margins.

the hot component alone. On the other hand, if the hot and warm components inferred from the ${ }^{12} \mathrm{CO}$ rotational diagram only reflect the presence of a temperature gradient in the same physical component, then we would infer much lower $\mathrm{H}_{2} \mathrm{O}_{\mathrm{h}} /\left(\mathrm{CO}_{\mathrm{h}}+\mathrm{CO}_{\mathrm{w}}\right) \simeq 0.02$ abundances (see Table 3 ). Such low $\mathrm{H}_{2} \mathrm{O}$ abundances are difficult to reconcile with non-dissociative $C$-shocks shielded from UV radiation and are more consistent with low-velocity $J$-shocks (see below).

Planar-shock model predictions of absolute $\mathrm{H}_{2} \mathrm{O}$ line intensities depend again on the unknown beam filling factor of the shocked material. Figure 10 shows a comparison of selected $\mathrm{H}_{2} \mathrm{O}$ line intensity ratios with recent unidimensional models of non-dissociative $C$-type and $J$-type shocks (the latter for $v_{\mathrm{s}}<30 \mathrm{~km} \mathrm{~s}^{-1}$ ) by Flower \& Pineau Des Forêts (2010). In order to avoid using optically thick line diagnostics, different beam dilutions and extinction corrections, we selected low opacity $p-\mathrm{H}_{2} \mathrm{O}$ lines arising from high energy levels $\left(E_{\mathrm{u}} / k \sim 300-650 \mathrm{~K}\right)$ observed at similar wavelengths. The upper panel shows the $3_{22}-2_{11} / 6_{06}-5_{15}$ line ratio $(89.988 / 83.284 \mu \mathrm{m})$ and the lower panel shows the $4_{31}-3_{22} / 4_{22}-3_{13}(56.325 / 57.637 \mu \mathrm{m})$ line ratio. The grey horizontal areas show the observed line intensity ratios and their error margins. From this comparison we conclude that lowvelocity, non-dissociative shocks $\left(v_{\mathrm{s}} \lesssim 20 \mathrm{~km} \mathrm{~s}^{-1}\right)$ with a preshock density of $n_{0} \simeq 2 \times 10^{5} \mathrm{~cm}^{-3}$ are needed to reproduce the observed ratios. Figure 10 favors small-scale non-dissociative $J$-shocks, although it is difficult to determine any possible contribution of $C$-type shocks in the frame of current shock models and lack of spectral/angular resolution at $\lambda<100 \mu \mathrm{m}$.

Besides, the very different shock velocities required by $\mathrm{Ne}^{+}$versus $\mathrm{H}_{2} \mathrm{O}$ lines suggests that both emissions arise from different locations. This will be the case if the $\mathrm{Ne}^{+}$emission arises from fast shocks within the jet itself, but the $\mathrm{H}_{2} \mathrm{O}$ (and $\mathrm{H}_{2}$ ) 
emission arises in lower velocity shocks along the inner walls of the outflow cavity. Finally, note that the similar excitation conditions in the hot and dense gas $\left(P / k=n T_{\mathrm{K}} \simeq 8 \times\right.$ $10^{9} \mathrm{~K} \mathrm{~cm}^{-3}$ ), as well as the similar spatial distribution of the nearby $\mathrm{CO} J=29-28$ and $p-\mathrm{H}_{2} \mathrm{O} 3_{22}-2_{11}$ lines shown in Fig. 4 suggests that the hot $\mathrm{CO}$ and the excited $\mathrm{H}_{2} \mathrm{O}$ lines arise in the same shock component.

The UV radiation field probed by the $[\mathrm{C}$ II $] 158 \mu \mathrm{m}$ line can also heat the gas along the outflow cavity walls (see e.g., Visser et al. 2012). UV photons will modify the chemistry of the shocked gas (e.g., by photodissociating $\mathrm{H}_{2} \mathrm{O}$ and enhancing the $\mathrm{OH}$ and $\mathrm{O}$ abundances; see Sect. 5.3), but in terms of gas heating, they are likely not a dominant heating mechanism of the hot gas emission seen towards low-mass protostars (see Karska et al. 2012). All in all, the relative line intensities of the detected atomic and molecular species confirm the presence of both fast dissociative and lower velocity, non-dissociative shocks that are irradiated by UV radiation fields.

\subsection{Line cooling}

Complete far-IR and submm spectral scans allow an unbiased determination of the line cooling in YSOs. Line photons following collisional excitation and escaping the region are responsible of the gas cooling. Hence, the observed line luminosities in a broadband spectral scan provide a good measurement of the total gas cooling budget (see Table 1). In Serpens SMM1, $\sim 54 \%$ of the total line luminosity is due to ${ }^{12} \mathrm{CO}$ lines, followed by $\mathrm{H}_{2} \mathrm{O}\left(\sim 22 \%\right.$ and $\left.L\left(\mathrm{H}_{2} \mathrm{O}\right) / L\left({ }^{12} \mathrm{CO}\right) \simeq 0.4\right)$, [O I] $(\sim 12 \%)$ and $\mathrm{OH}(9 \%)$. The total far-IR and submm line luminosity $L_{\mathrm{FIR} \mathrm{SMM}}=L\left({ }^{12} \mathrm{CO}\right)+L\left(\mathrm{H}_{2} \mathrm{O}\right)+L(\mathrm{O} \mathrm{I})+L(\mathrm{OH})+L\left({ }^{13} \mathrm{CO}\right)+L\left(\mathrm{C}^{+}\right)$ is $L_{\mathrm{FIR} \mathrm{SMM}} \simeq 0.12 L_{\odot}$, and the ratio $L_{\mathrm{mol}} / L_{\mathrm{bol}}$ between the molecular line luminosity, $L_{\mathrm{mol}}=L\left({ }^{12} \mathrm{CO}\right)+L\left(\mathrm{H}_{2} \mathrm{O}\right)+L(\mathrm{OH})$, and the bolometric luminosity is $\simeq 3.5 \times 10^{-3}$, all consistent with the expected emission of a Class 0 source (see e.g., Giannini et al. 2001). Note that only if the extinction in the mid-IR is larger than $A_{V} \simeq 200$, then the intrinsic luminosity of the observed $\mathrm{H}_{2}$ lines will be higher than those of $\mathrm{CO}$ and $\mathrm{H}_{2} \mathrm{O}$.

In our model, $\mathrm{CO}$ dominates the line cooling (Table 1) with more than half of the total CO luminosity arising from the warm component (a mixture of shocks and UV-illuminated material) and with a warm/hot luminosity ratio of $L\left(\mathrm{CO}_{\mathrm{w}}\right) / L\left(\mathrm{CO}_{\mathrm{h}}\right) \simeq 5$. $\mathrm{H}_{2} \mathrm{O}$ is the second most important species, with a dominant contribution to the hot shocked gas cooling. Note that we predict that $\gtrsim 80 \%$ of the $\mathrm{H}_{2} \mathrm{O}$ line luminosity is radiated in the $55 \mu \mathrm{m}<\lambda<200 \mu \mathrm{m}$ range and only $\sim 5 \%$ at shorter wavelengths (in agreement with the absence of strong $\mathrm{H}_{2} \mathrm{O}$ lines in the Spitzer observations).

$[\mathrm{O} \mathrm{I}]$ and $\mathrm{OH}$ lines also contribute to the gas cooling. In particular, both the absolute $\mathrm{OH}$ luminosities and the observed [OI]63 $\mu \mathrm{m} / \mathrm{H}_{2} 0-0 \mathrm{~S}(1) \gg 1$ intensity ratio are too bright for non-dissociative $C$-shock models (Kaufman \& Neufeld 1996; Flower \& Pineau Des Forêts 2010). As noted before, a significant fraction of the $[\mathrm{O} \mathrm{I}]$ and $\mathrm{OH}$ line emission likely contributes to the cooling behind a fast, dissociative $J$-type shock.

\subsection{Shock and UV-driven chemistry}

The plethora of atomic fine-structure lines and to a lesser extent, the relatively high $\mathrm{OH} / \mathrm{H}_{2} \mathrm{O} \lesssim 0.5$ abundance ratio inferred in the warm+hot component, confirms the presence of strong dissociative shocks in the inner envelope. In addition, owing to efficient $\mathrm{H}_{2} \mathrm{O}$ photodissociation, an enhanced UV radiation field illuminating the shocked gas can produce a high $\mathrm{OH} / \mathrm{H}_{2} \mathrm{O}$ abundance ratios (see Goicoechea et al. 2011, in the
Orion Bar PDR). The UV radiation field near low-mass protostars (roughly a diluted $<10000 \mathrm{~K}$ blackbody) is thought to be dominated by Ly $\alpha$ photons $(10.2 \mathrm{eV})$ arising from accreting material onto the star (Bergin et al. 2003) and from fast dissociative $J$-shocks (Hollenbach \& McKee 1979) such as those inferred in Serpens SMM1 (Sect. 5.1.1). Ly $\alpha$ radiation can dissociate $\mathrm{H}_{2} \mathrm{O}$ (producing enhanced $\mathrm{OH}$ and $\mathrm{O}$ ) but can not dissociate CO $(\sim 11.1 \mathrm{eV})$ nor ionize sulfur $(10.4 \mathrm{eV})$ or carbon $(11.3 \mathrm{eV})$ atoms. However, if significant $\mathrm{C}^{+}$and $\mathrm{H}_{2}$ are present, the ion-neutral drift in $C$-type shocks would significantly enhance the $\mathrm{CH}^{+}$formation rate compared to $J$-type shocks (see Falgarone et al. 2010, for the detection of $\mathrm{CH}^{+} J=1-0$ broad outflow wings in the shock associated with the DR 21 massive star forming region). The lack of $\mathrm{CH}^{+} J=3-2$ emission in Serpens SMM1 provides an upper limit for the $\mathrm{CH}^{+}$column density in the shocked gas $\left(\mathrm{CH}^{+} / \mathrm{H}_{2} \mathrm{O}<0.01\right)$ and will help to constrain UV-irradiated shocks models (Kaufman et al., in prep.; Lesaffre et al., in prep.). The $\mathrm{CH}^{+} J=3-2$ line is detected in PDRs illuminated by massive OB stars where $\mathrm{C}^{+}$ is the dominant carbon species (Goicoechea et al. 2011; Nagy et al., in prep.) and, as in DR 21, the UV radiation field contains photons with energies higher than Ly $\alpha$ (up to $13.6 \mathrm{eV}$ ). Excited lines from reactive ions such as $\mathrm{CO}^{+}$(that forms by reaction of $\mathrm{C}^{+}$with $\mathrm{OH}$ ) and are related with the presence of strong UV radiation fields in the shocked gas, are not detected in the PACS spectrum despite previous tentative assignations of several high-energy far-IR lines (see Ceccarelli et al. 1997, for IRAS 16293-2422)

The spatial distribution of the [C II] $158 \mu$ m line emission detected towards Serpens SMM1 (Fig. 4) is similar to that of other species in the outflow (with brighter emission in the outflow position than towards the protostar itself). The detection of faint $\mathrm{C}^{+}$emission indicates the presence of a relatively weak UV field (but able to ionize $\mathrm{C}$ atoms and dissociate $\mathrm{CO}$ ) along the outflow. Besides, the increase of the $\mathrm{HCO}^{+} / \mathrm{HCN}$ abundance ratio in the warm temperature component (HCN is easily photodissociated while $\mathrm{HCO}^{+}$is abundant in the dense gas directly exposed to strong UV radiation) is another signature of UV photons. Indeed, the mere detection of [C I] $370,609 \mu \mathrm{m}$ lines at the poor spectral resolution of the SPIRE-FTS towards the protostar shows that $[\mathrm{CI}]$ lines are significantly brighter than towards $\mathrm{HH} 46$ (van Kempen et al. 2009) or NGC 1333 IRAS 4A/4B (Yildiz et al. 2012). The presence of weak $C^{+}$line emission and of Ly $\alpha$ radiation in protostars like Serpens SMM1 (although difficult to detect) suggests that UV-irradiated shocks are a common phenomenon in YSOs.

X-ray emission is also expected in low-mass YSOs (Stäuber et al. 2006, 2007; Feigelson 2010) and they produce internally generated UV radiation fields after collisions of energetic photoelectrons with $\mathrm{H}$ and $\mathrm{H}_{2}$ (Maloney et al. 1996; Hollenbach \& Gorti 2009). Although X-ray detections with Chandra have been reported towards the nearby Serpens SMM5, SMM6 and S68Nb protostars (Winston et al. 2007), the strength of any $\mathrm{X}$-ray emission from Serpens SMM1 is unknown, possibly because of the high column density to the embedded source.

High sensitivity and velocity-resolved observations of $\mathrm{CH}^{+}$, $\mathrm{CO}^{+}, \mathrm{SO}^{+}$and other reactive ions related with the presence of ionized atoms (e.g., $\mathrm{C}^{+}$and $\mathrm{S}^{+}$) will help us to characterize these UV-irradiated shocks in more detail.

\subsection{Comparison with other low-mass protostars}

Comparative spectroscopy of protostars in different stages of evolution allows us to identify the common and the more 
peculiar processes associated with the first stages of star formation. Comparing the far-IR spectrum of the NGC 1333 IRAS 4B outflow to that of the Serpens SMM1 protostar, both show similar high $\mathrm{H}_{2} \mathrm{O}$ luminosities $\left(L\left(\mathrm{H}_{2} \mathrm{O}\right) \sim\right.$ $\left.0.03 L_{\odot}\right)$. However, Serpens SMM1 shows a factor $\sim 50$ stronger [OI] luminosity and a lower $L\left(\mathrm{H}_{2} \mathrm{O}\right) / L(\mathrm{CO})$ ratio. The weak [OI] emission and high $\mathrm{H}_{2} \mathrm{O}$ luminosity in IRAS 4B outflow $\left(L\left(\mathrm{H}_{2} \mathrm{O}\right) / L(\mathrm{CO}) \simeq 1\right)$ has been interpreted as non-dissociative $C$-shocks shielded from UV radiation (Herczeg et al. 2012). Indeed, $\mathrm{C}^{+}$is not detected in IRAS 4B outflow whereas it is detected in Serpens SMM1 protostar and outflow (see Fig. 4). On the other hand, the strong [OI] and $\mathrm{OH}$ emission towards the Serpens SMM1 protostar itself is more similar to that of Class I source HH46 (van Kempen et al. 2010) where a $L(\mathrm{OH}) / L\left(\mathrm{H}_{2} \mathrm{O}\right) \simeq 0.5$ luminosity ratio has been inferred (vs. $\sim 0.4$ in SMM1). One possibility is that a high-velocity jet impinging on the dense inner envelope produces dissociative $J$-shocks (van Kempen et al. 2010) and enhanced [O I] and $\mathrm{OH}$ emission compared to non-dissociative $C$-shocks. Although they could not distinguish the dominant scenario, either $J$-shocks or UVirradiated $C$-shocks have been also proposed to explain the $\mathrm{OH}$ emission seen in the high-mass YSO W3 IRS 5 (Wampfler et al. 2011).

The detection of [Ne II], [Fe II], [Si II] and [S I] fine-structure lines towards Serpens SMM1 reinforces the scenario of both fast and slow shocks as well as UV radiation near the protostar, however, it is difficult to extract the exact geometry of the different shock components in the circumstellar environment (e.g., $\mathrm{Ne}^{+}$versus $\mathrm{H}_{2} \mathrm{O}$ line emitting regions). Note that the presence of an embedded jet in the Class 0 source L1448 was reported from the detection of [Fe II] and [Si II] lines by Dionatos et al. (2009), and the same lines have been detected towards the nearby YSOs Serpens SMM3 and SMM4 (Lahuis et al. 2010). However, they did not detect the [Ne II] $12.8 \mu \mathrm{m}$ line that requires fast shock velocities (Lahuis et al. 2007; Baldovin-Saavedra et al. 2012) or X-ray radiation (see Güdel et al. 2010, for Class II disk sources).

Compared to HH46, CO lines with $J_{\mathrm{u}}>30$ and higher excitation $\mathrm{H}_{2} \mathrm{O}$ lines are detected in Serpens SMM1 (not necessarily due to different excitation conditions but maybe just because lines are much brighter in SMM1). We have proposed that this hot $\mathrm{CO}$ and $\mathrm{H}_{2} \mathrm{O}$ emission arises from low velocity, nondissociative shocks in the inner walls of the outflow cavity compressing the gas to very high thermal pressures $\left(P / k=n T_{\mathrm{K}} \simeq\right.$ $10^{9-10} \mathrm{~K} \mathrm{~cm}^{-3}$ ). Based on the large gas compression factors and $\mathrm{H}_{2} \mathrm{O}$ line profiles seen toward several shock spots in bipolar outflows (far from the protostellar sources) Santangelo et al. (2012) and Tafalla et al. (in prep.) conclude that current low velocity $J$-shocks models explain their observations better than stationary $C$-shocks models. New shock models with a more detailed geometrical description and including the effects of UV radiation are clearly needed to determine the exact nature of the shocks inferred in the dense circumstellar gas near protostars.

The warm CO line emission $\left(15 \lesssim J_{\mathrm{u}} \lesssim 25\right)$ observed in Serpens SMM1 is a common feature in the far-IR spectrum of most protostars even if they are in different stages of evolution. This was previously observed by ISO (e.g., Giannini et al. 2001) and now by Herschel (e.g., Karska et al., submitted.; Green et al., in prep.; Manoj et al., in prep.). This $\mathrm{CO}$ emission ubiquity necessarily means that a broad combination of shock velocities and densities can produce a similar warm CO spectrum in different low- and high-mass YSOs.

The $L(\mathrm{OH}) / L\left(\mathrm{H}_{2} \mathrm{O}\right), L(\mathrm{CO}) / L([\mathrm{O} \mathrm{I}])$ and $L\left(\mathrm{H}_{2} \mathrm{O}\right) / L([\mathrm{O} \mathrm{I}])$ luminosity ratios in Serpens SMM1 have intermediate values between those in NGC 1333 IRAS 4B Class 0 YSO and HH46 Class I YSO (with ratios closer to SMM1) suggesting that the different luminosity ratios in IRAS 4B outflow are either a consequence of an earlier stage of evolution, or simply because the IRAS $4 \mathrm{~B}$ outflow is peculiar, with very high $\mathrm{H}_{2} \mathrm{O}$ abundances not affected by UV photodissociation. Indeed, one of the main conclusions of all these studies is that water vapour lines are a roboust probe of shocked gas in protostellar environments. In Serpens SMM1 we infer a total $\mathrm{H}_{2} \mathrm{O}_{\mathrm{h}} /\left(\mathrm{CO}_{\mathrm{h}}+\mathrm{CO}_{\mathrm{w}}\right) \simeq 0.02$ abundance ratio in the warm + hot components traced by our far-IR observations. Only if the hot component was an independent physical component, the $\mathrm{H}_{2} \mathrm{O}$ abundances would be much higher $\left(\mathrm{H}_{2} \mathrm{O}_{\mathrm{h}} / \mathrm{CO}_{\mathrm{h}} \simeq 0.4\right)$. However, this value is higher than the typical $\mathrm{H}_{2} \mathrm{O} / \mathrm{CO}$ abundance ratios inferred from HIFI observations of the ground-state $o-\mathrm{H}_{2} \mathrm{O} 1_{10}-1_{01}$ line (at $\sim 557 \mathrm{GHz}$ ) towards a large sample of low-mass protostars (Kristensen et al. 2012). In these velocityresolved observations, the $\mathrm{H}_{2} \mathrm{O} / \mathrm{CO}$ abundance ratio increases from $\sim 10^{-3}$ at low outflow velocities $\left(<5 \mathrm{~km} \mathrm{~s}^{-1}\right)$ to $\sim 0.1$ at high outflow velocities $\left(>10 \mathrm{~km} \mathrm{~s}^{-1}\right)$. Therefore, our inferred abundance ratio of $\mathrm{H}_{2} \mathrm{O}_{\mathrm{h}} /\left(\mathrm{CO}_{\mathrm{h}}+\mathrm{CO}_{\mathrm{w}}\right) \simeq 0.02$ from PACS observations seems a better description of the water vapour abundance in the protostellar shocked gas. Besides, it is also consistent with recent determinations from velocity-resolved HIFI observations of moderately excited $\mathrm{H}_{2} \mathrm{O}$ lines $\left(E_{\mathrm{u}} / k<215 \mathrm{~K}\right)$ (see e.g., Santangelo et al. 2012, for L1448 outflow).

\section{Summary and conclusions}

We have presented the first complete far-IR and submm spectrum of a Class 0 protostar (Serpens SMM1) taken with Herschel in the $\sim 55-671 \mu \mathrm{m}$ window. The data are complemented with mid-IR spectra in the $\sim 10-37 \mu \mathrm{m}$ window retrieved from the Spitzer archive and first discussed here. These spectroscopic observations span almost 2 decades in wavelength and allow us to unveil the most important heating and chemical processes associated with the first evolutionary stages of low-mass protostars. In particular, we obtained the following results:

- Serpens SMM1 shows a very rich far-IR and submm emission spectrum, with more than 145 lines, most of them rotationally excited lines of ${ }^{12} \mathrm{CO}$ (up to $J=42-41$ ), $\mathrm{H}_{2} \mathrm{O}$, $\mathrm{OH},{ }^{13} \mathrm{CO}, \mathrm{HCN}, \mathrm{HCO}^{+}$and weaker emission from [C II] 158 and [CI]370, $609 \mu \mathrm{m}$. [OI]63 $\mu \mathrm{m}$ is the brightest line. Approximately half of the total far-IR and submm line luminosity is provided by $\mathrm{CO}$ rotational emission, with important contribution from $\mathrm{H}_{2} \mathrm{O}(\sim 22 \%)$, [O I] $(\sim 12 \%)$ and $\mathrm{OH}(\sim 9 \%)$.

- The mid-IR spectrum shows many fewer lines (probably due to severe dust extinction and lack of spectral resolution). Bright fine structure lines from $\mathrm{Ne}^{+}, \mathrm{Fe}^{+}, \mathrm{Si}^{+}$, and $\mathrm{S}$, as well as weak $\mathrm{H}_{2} \mathrm{~S}(1)$ and $\mathrm{S}(2)$ pure rotational lines are detected.

- The ${ }^{12} \mathrm{CO}$ rotational diagram suggests the presence of 3 temperature components with $T_{\text {rot }}^{\text {hot }} \simeq 620 \pm 30 \mathrm{~K}$, $T_{\text {rot }}^{\text {warm }} \simeq 340 \pm 40 \mathrm{~K}$ and $T_{\text {rot }}^{\text {cool }} \simeq 100 \pm 15 \mathrm{~K}$. As predicted by SED models in the literature, the detection of $\mathrm{H}_{2} \mathrm{O}, \mathrm{OH}$, $\mathrm{HCO}^{+}$and $\mathrm{HCN}$ emission lines from very high critical density transitions suggests that the density in the inner envelope is high $\left(n\left(\mathrm{H}_{2}\right) \gtrsim 5 \times 10^{6} \mathrm{~cm}^{-3}\right)$, and thus the CO rotational temperatures provide a good approximation to the gas temperature $\left(T_{\text {rot }} \lesssim T_{\mathrm{k}}\right)$.

- A non-LTE, multi-component model allowed us to approximately quantify the contribution of the different temperature components $\left(T_{\mathrm{k}}^{\mathrm{hot}} \approx 800 \mathrm{~K}, T_{\mathrm{k}}^{\mathrm{warm}} \approx 375 \mathrm{~K}\right.$ and $T_{\mathrm{k}}^{\mathrm{cool}} \approx$ $150 \mathrm{~K}$ ) and to estimate relative chemical abundances. The detected mid-IR $\mathrm{H}_{2}$ lines provide a lower limit to $\mathrm{H}_{2}$ column 
density of $N_{\mathrm{H}_{2}} \geq 10^{22} \mathrm{~cm}^{-2}$. We derive the following upper limit abundances with respect to $\mathrm{H}_{2}$ in the hot+warm components: $x(\mathrm{CO}) \leq 10^{-4}, x\left(\mathrm{H}_{2} \mathrm{O}\right) \leq 0.5 \times 10^{-5}$ and $x(\mathrm{OH}) \leq 10^{-6}$. The inferred water vapour abundance is higher than the value typically found in cold interstellar clouds but lower than that expected in hot shocked gas completely shielded from UV radiation.

- Excited $\mathrm{CO}, \mathrm{H}_{2} \mathrm{O}$ and $\mathrm{OH}$ emission lines arising from high energy levels are detected (up to $E_{\mathrm{u}} / k=4971 \mathrm{~K}, E_{\mathrm{u}} / k=$ $1036 \mathrm{~K}$ and $E_{\mathrm{u}} / k=618 \mathrm{~K}$ respectively). These species arise in the hot+warm gas $\left(M \lesssim 0.03 M_{\odot}\right)$ that we associate with a mixture of shocks. The observed hot $\mathrm{CO}, \mathrm{H}_{2} \mathrm{O}$ and $\mathrm{H}_{2}$ lines provide a lower limit to the gas temperature of $\sim 700 \mathrm{~K}$. The excited $\mathrm{H}_{2} \mathrm{O}$ line emission is consistent with detailed model predictions of low-velocity non-dissociative shocks (with $v_{\mathrm{s}} \lesssim 20 \mathrm{~km} \mathrm{~s}^{-1}$ ).

- Fast dissociative (and ionizing) shocks with velocities $v_{\mathrm{s}}>$ $60 \mathrm{~km} \mathrm{~s}^{-1}$ and pre-shock densities $\geq 10^{4} \mathrm{~cm}^{-3}$ related with the presence of an embedded atomic jet are needed to explain the observed $\mathrm{Ne}^{+}, \mathrm{Fe}^{+}, \mathrm{Si}^{+}$and $\mathrm{S}$ fine-structure emission and also the bright and velocity-shifted [O I] $63 \mu \mathrm{m}$ line emission. Dissociative $J$-shocks produced by the jet impacting the ambient material are the most probable origin of the bright [O I] and $\mathrm{OH}$ emission and of a significant fraction of the warm $\mathrm{CO}$ emission. In addition, water vapour photodissociation in UV-irradiated non-dissociative shocks along the outflow cavity walls can also contribute to the [O I] and $\mathrm{OH}$ emission.

Compared to other protostars, the large number of lines detected towards Serpens SMM1 reveals the great complexity of the protostellar environment. Both fast and slow shocks are needed to explain the presence of atomic fine structure lines and high excitation molecular lines. The spectra also show the signature of $\mathrm{UV}$ radiation $\left(\mathrm{C}^{+}\right.$and $\mathrm{C}$ towards the protostar and outflow). Therefore, most species (including $\mathrm{H}_{2} \mathrm{O}, \mathrm{OH}, \mathrm{O}$ and trace molecules such as $\mathrm{CH}^{+}$) arise in shocked gas illuminated by UV (even X-ray) radiation fields. Irradiated shocks are likely a very common phenomenon in YSOs.

Acknowledgements. We acknowledge our WISH internal referees, D. Neufeld and P. Bjerkeli for very helpful comments on an earlier version of the manuscript. We also thank the entire WISH team for many useful and vivid discussions in the last years. We finally thank the anonymous referee and M. Walmsley for useful comments. WISH research in Leiden is supported by the Netherlands Research School for Astronomy (NOVA), by a Spinoza grant and grant 614.001.008 from the Netherlands Organisation for Scientific Research (NWO), and by EU-FP7 grant 238258 (LASSIE). J.R.C., J.C. and M.E. thank the Spanish MINECO for funding support through grants AYA2009-07304 and CSD2009-00038. J.R.G. is supported by a Ramón y Cajal research contract from the MINECO and cofinanced by the European Social Fund.

\section{References}

Bachiller, R., \& Tafalla, M. 1999, in The Origin of Stars and Planetary Systems, eds. C. J. Lada, \& N. D. Kylafis, NATO ASIC Proc., 540, 227

Baldovin-Saavedra, C., Audard, M., Carmona, A., et al. 2012, A\&A, 543, A30 Bergin, E., Calvet, N., D’Alessio, P., \& Herczeg, G. J. 2003, ApJ, 591, L159

Bontemps, S., Andre, P., Terebey, S., \& Cabrit, S. 1996, A\&A, 311, 858

Caselli, P., Keto, E., Pagani, L., et al. 2010, A\&A, 521, L29

Ceccarelli, C., Caux, E., Wolfire, M., et al. 1997, in The first ISO workshop on Analytical Spectroscopy, eds. A. M. Heras, K. Leech, N. R. Trams, \& M. Perry, ESA SP, 419, 43
Cernicharo, J. 2012, in Proc. of the European Conference on Laboratory Astrophysics, eds. C. Stehlé, C. Joblin, \& L. d'Hendecourt, Eur. Astron. Soc. Publ. Ser., 2012, 4

Cernicharo, J., Goicoechea, J. R., Daniel, F., et al. 2006, ApJ, 649, L33

Chiar, J. E., Ennico, K., Pendleton, Y. J., et al. 2007, ApJ, 666, L73

Davis, C. J., Matthews, H. E., Ray, T. P., Dent, W. R. F., \& Richer, J. S. 1999, MNRAS, 309, 141

Dionatos, O., Nisini, B., Garcia Lopez, R., et al. 2009, ApJ, 692, 1

Dionatos, O., Nisini, B., Codella, C., \& Giannini, T. 2010, A\&A, 523, A29

Draine, B. T., \& McKee, C. F. 1993, ARA\&A, 31, 373

Dzib, S., Loinard, L., Mioduszewski, A. J., et al. 2010, ApJ, 718, 610

Eiroa, C., Djupvik, A. A., \& Casali, M. M. 2008, The Serpens Molecular Cloud, ed. B. Reipurth, 693

Enoch, M. L., Corder, S., Dunham, M. M., \& Duchêne, G. 2009, ApJ, 707, 103

Evans, II, N. J., Dunham, M. M., Jørgensen, J. K., et al. 2009, ApJS, 181, 321

Falgarone, E., Ossenkopf, V., Gerin, M., et al. 2010, A\&A, 518, L118

Feigelson, E. D. 2010, Proc. National Academy of Science, 107, 7153

Flower, D. R., \& Pineau Des Forêts, G. 2010, MNRAS, 406, 1745

Giannini, T., Nisini, B., \& Lorenzetti, D. 2001, ApJ, 555, 40

Goicoechea, J. R., \& Cernicharo, J. 2002, ApJ, 576, L77

Goicoechea, J. R., Cernicharo, J., Lerate, M. R., et al. 2006, ApJ, 641, L49

Goicoechea, J. R., Joblin, C., Contursi, A., et al. 2011, A\&A, 530, L16

Griffin, M. J., Abergel, A., Abreu, A., et al. 2010, A\&A, 518, L3

Güdel, M., Lahuis, F., Briggs, K. R., et al. 2010, A\&A, 519, A113

Herczeg, G. J., Karska, A., Bruderer, S., et al. 2012, A\&A, 540, A84

Hogerheijde, M. R., van Dishoeck, E. F., Salverda, J. M., \& Blake, G. A. 1999, ApJ, 513, 350

Hollenbach, D., \& Gorti, U. 2009, ApJ, 703, 1203

Hollenbach, D., \& McKee, C. F. 1979, ApJS, 41, 555

Hollenbach, D., \& McKee, C. F. 1989, ApJ, 342, 306

Houck, J. R., Roellig, T. L., van Cleve, J., et al. 2004, ApJS, 154, 18

Karska, A., Herczeg, G. J., van Dishoeck, E. F., et al. 2012, A\&A, submitted

Kaufman, M. J., \& Neufeld, D. A. 1996, ApJ, 456, 611

Kristensen, L. E., van Dishoeck, E. F., Bergin, E. A., et al. 2012, A\&A, 542, A8

Lahuis, F., van Dishoeck, E. F., Blake, G. A., et al. 2007, ApJ, 665, 492

Lahuis, F., van Dishoeck, E. F., Jørgensen, J. K., Blake, G. A., \& Evans, N. J. 2010, A\&A, 519, A3

Langer, W. D., \& Penzias, A. A. 1990, ApJ, 357, 477

Laor, A., \& Draine, B. T. 1993, ApJ, 402, 441

Larsson, B., Liseau, R., Men'shchikov, A. B., et al. 2000, A\&A, 363, 253

Larsson, B., Liseau, R., \& Men'shchikov, A. B. 2002, A\&A, 386, 1055

Maloney, P. R., Hollenbach, D. J., \& Tielens, A. G. G. M. 1996, ApJ, 466, 561

Melnick, G. J., Tolls, V., Neufeld, D. A., et al. 2008, ApJ, 683, 876

Neufeld, D. A., \& Dalgarno, A. 1989, ApJ, 340, 869

Neufeld, D. A., Melnick, G. J., Sonnentrucker, P., et al. 2006, ApJ, 649, 816

Nisini, B., Bacciotti, F., Giannini, T., et al. 2005, A\&A, 441, 159

Offer, A. R., \& van Dishoeck, E. F. 1992, MNRAS, 257, 377

Pilbratt, G. L., Riedinger, J. R., Passvogel, T., et al. 2010, A\&A, 518, L1

Poglitsch, A., Waelkens, C., Geis, N., et al. 2010, A\&A, 518, L2

Rodríguez, L. F., Loinard, L., D’Alessio, P., Wilner, D. J., \& Ho, P. T. P. 2005, ApJ, 621, L133

Santangelo, G., Nisini, B., Giannini, T., et al. 2012, A\&A, 538, A45

Smith, J. D. T., Armus, L., Dale, D. A., et al. 2007, PASP, 119, 1133

Stäuber, P., Jørgensen, J. K., van Dishoeck, E. F., Doty, S. D., \& Benz, A. O. 2006, A\&A, 453, 555

Stäuber, P., Benz, A. O., Jørgensen, J. K., et al. 2007, A\&A, 466, 977

van Dishoeck, E. F., Kristensen, L. E., Benz, A. O., et al. 2011, PASP, 123, 138

van Kempen, T. A., van Dishoeck, E. F., Hogerheijde, M. R., \& Güsten, R. 2009, A\&A, 508, 259

van Kempen, T. A., Kristensen, L. E., Herczeg, G. J., et al. 2010, A\&A, 518, L121

Visser, R., Kristensen, L. E., Bruderer, S., et al. 2012, A\&A, 537, A55

Walmsley, M., Pineau des Forêts, G., \& Flower, D. 2005, in Astrochemistry: Recent Successes and Current Challenges, eds. D. C. Lis, G. A. Blake, \& E. Herbst, IAU Symp., 231, 135

Wampfler, S. F., Bruderer, S., Kristensen, L. E., et al. 2011, A\&A, 531, L16

Wampfler, S. F., Bruderer, S., Karska, A., et al. 2012, A\&A, submitted

Watson, D. M., Bohac, C. J., Hull, C., et al. 2007, Nature, 448, 1026

Whittet, D. C. B. 2003, Dust in the galactic environment

Winston, E., Megeath, S. T., Wolk, S. J., et al. 2007, ApJ, 669, 493

Yıldız, U. A., Kristensen, L. E., van Dishoeck, E. F., et al. 2012, A\&A, 542, A86

Pages 15 to 17 are available in the electronic edition of the journal at http://www . aanda. org 
J. R. Goicoechea et al.: The complete spectrum of the Class 0 protostar Serpens SMM1

\section{Appendix A: Complementary figures and tables}

Table A.1. List of detected lines towards Serpens SMM1 and line fluxes from SPIRE and PACS central spaxels (corrected for extended emission in the case of PACS).

\begin{tabular}{|c|c|c|c|c|}
\hline Species & Transition & $\begin{array}{l}E_{\mathrm{up}} \\
(\mathrm{K}) \\
\end{array}$ & $\begin{array}{c}\lambda \\
(\mu \mathrm{m})\end{array}$ & $\begin{array}{l}\text { Line flux } \\
\left(\mathrm{W} \mathrm{m}^{-2}\right)\end{array}$ \\
\hline $\mathrm{HCO}^{+}$ & $5-4$ & 64.2 & 672.326 & $2.24 \mathrm{E}-16$ \\
\hline $\mathrm{CO}$ & $4-3$ & 55.3 & 650.252 & $4.11 \mathrm{E}-16$ \\
\hline $\mathrm{C}$ & ${ }^{3} \mathrm{P}_{1}-{ }^{3} \mathrm{P}_{0}$ & 23.6 & 609.133 & $8.69 \mathrm{E}-17$ \\
\hline $\mathrm{HCO}^{+}$ & $6-5$ & 89.9 & 560.294 & $3.16 \mathrm{E}-17$ \\
\hline${ }^{13} \mathrm{CO}$ & $5-4$ & 79.3 & 544.161 & $6.33 \mathrm{E}-17$ \\
\hline $\mathrm{H}_{2} \mathrm{O}$ & $1_{10}-1_{01}$ & 26.7 & 538.289 & $4.96 \mathrm{E}-17$ \\
\hline $\mathrm{CO}$ & $5-4$ & 83.0 & 520.231 & $5.13 \mathrm{E}-16$ \\
\hline $\mathrm{HCO}^{+}$ & $7-6$ & 119.8 & 480.275 & $6.20 \mathrm{E}-17$ \\
\hline${ }^{13} \mathrm{CO}$ & $6-5$ & 111.1 & 453.498 & $1.11 \mathrm{E}-16$ \\
\hline $\mathrm{CO}$ & $6-5$ & 116.2 & 433.556 & $6.85 \mathrm{E}-16$ \\
\hline $\mathrm{HCN}$ & $8-7$ & 153.1 & 422.912 & $3.28 \mathrm{E}-17$ \\
\hline $\mathrm{HCO}^{+}$ & $8-7$ & 154.1 & 420.263 & $5.51 \mathrm{E}-17$ \\
\hline $\mathrm{H}_{2} \mathrm{O}$ & $2_{11}-2_{02}$ & 136.9 & 398.643 & $1.67 \mathrm{E}-16$ \\
\hline${ }^{13} \mathrm{CO}$ & $7-6$ & 148.1 & 388.743 & $1.42 \mathrm{E}-16$ \\
\hline $\mathrm{HCO}^{+}$ & $9-8$ & 192.6 & 373.591 & $4.10 \mathrm{E}-17$ \\
\hline $\mathrm{CO}$ & $7-6$ & 154.9 & 371.650 & $1.32 \mathrm{E}-15$ \\
\hline $\mathrm{C}$ & ${ }^{3} \mathrm{P}_{2}-{ }^{3} \mathrm{P}_{1}$ & 62.5 & 370.414 & $1.42 \mathrm{E}-16$ \\
\hline${ }^{13} \mathrm{CO}$ & $8-7$ & 190.4 & 340.181 & 4.16E-17 \\
\hline $\mathrm{HCO}^{+}$ & $10-9$ & 235.4 & 336.255 & $3.37 \mathrm{E}-17$ \\
\hline $\mathrm{CO}$ & $8-7$ & 199.1 & 325.225 & $1.68 \mathrm{E}-15$ \\
\hline $\mathrm{HCN}$ & $11-10$ & 280.7 & 307.641 & $5.55 \mathrm{E}-17$ \\
\hline $\mathrm{HCO}^{+}$ & $11-10$ & 282.4 & 305.712 & $8.91 \mathrm{E}-17$ \\
\hline $\mathrm{H}_{2} \mathrm{O}$ & $2_{02}-1_{11}$ & 100.8 & 303.459 & $4.40 \mathrm{E}-16$ \\
\hline${ }^{13} \mathrm{CO}$ & $9-8$ & 237.9 & 302.415 & $1.59 \mathrm{E}-16$ \\
\hline $\mathrm{CO}$ & $9-8$ & 248.9 & 289.120 & $1.54 \mathrm{E}-15$ \\
\hline $\mathrm{HCO}^{+}$ & $12-11$ & 333.8 & 280.257 & $8.18 \mathrm{E}-17$ \\
\hline $\mathrm{H}_{2} \mathrm{O}$ & $3_{12}-3_{03}$ & 215.2 & 273.193 & 3.23E- 16 \\
\hline${ }^{13} \mathrm{CO}$ & $10-9$ & 290.8 & 272.205 & $5.62 \mathrm{E}-17$ \\
\hline $\mathrm{H}_{2} \mathrm{O}$ & $1_{11}-0_{00}$ & 53.4 & 269.273 & $4.41 \mathrm{E}-16$ \\
\hline $\mathrm{CO}$ & $10-9$ & 304.2 & 260.240 & $2.02 \mathrm{E}-15$ \\
\hline $\mathrm{H}_{2} \mathrm{O}$ & $3_{21}-3_{12}$ & 271.0 & 257.790 & $2.48 \mathrm{E}-16$ \\
\hline${ }^{13} \mathrm{CO}$ & $11-10$ & 348.9 & 247.490 & $1.51 \mathrm{E}-16$ \\
\hline $\mathrm{H}_{2} \mathrm{O}$ & $2_{20}-2_{11}$ & 195.9 & 243.972 & $2.92 \mathrm{E}-17$ \\
\hline $\mathrm{CO}$ & $11-10$ & 365.0 & 236.613 & $2.17 \mathrm{E}-15$ \\
\hline${ }^{13} \mathrm{CO}$ & $12-11$ & 412.4 & 226.898 & 7.67E-17 \\
\hline $\mathrm{CO}$ & $12-11$ & 431.3 & 216.927 & $2.45 \mathrm{E}-15$ \\
\hline${ }^{13} \mathrm{CO}$ & $13-12$ & 481.0 & 209.476 & $1.51 \mathrm{E}-16$ \\
\hline $\mathrm{CO}$ & $13-12$ & 503.6 & 200.272 & $2.60 \mathrm{E}-15$ \\
\hline${ }^{13} \mathrm{CO}$ & $14-13$ & 555.0 & 194.546 & $1.32 \mathrm{E}-16$ \\
\hline $\mathrm{H}_{2} \mathrm{O}$ & $4_{13}-4_{04}$ & 396.4 & 187.110 & $5.74 \mathrm{E}-17$ \\
\hline $\mathrm{CO}$ & $14-13$ & 581.0 & 185.999 & $1.55 \mathrm{E}-15$ \\
\hline${ }^{13} \mathrm{CO}$ & $15-14$ & 634.2 & 181.608 & $6.57 \mathrm{E}-17$ \\
\hline $\mathrm{H}_{2} \mathrm{O}$ & $2_{21}-2_{12}$ & 159.9 & 180.488 & $5.25 \mathrm{E}-16$ \\
\hline $\mathrm{H}_{2} \mathrm{O}$ & $2_{12}-1_{01}$ & 80.1 & 179.527 & $9.72 \mathrm{E}-16$ \\
\hline $\mathrm{H}_{2} \mathrm{O}$ & $3_{03}-2_{12}$ & 162.5 & 174.626 & $1.21 \mathrm{E}-15$ \\
\hline $\mathrm{CO}$ & $15-14$ & 663.9 & 173.631 & $2.20 \mathrm{E}-15$ \\
\hline${ }^{13} \mathrm{CO}$ & $16-15$ & 718.7 & 170.290 & $3.63 \mathrm{E}-17$ \\
\hline $\mathrm{OH}$ & ${ }^{2} \Pi_{1 / 2} J=3 / 2^{-}-1 / 2^{+}$ & 269.8 & 163.396 & $1.92 \mathrm{E}-16$ \\
\hline $\mathrm{OH}$ & ${ }^{2} \Pi_{1 / 2} J=3 / 2^{+}-1 / 2^{-}$ & 270.2 & 163.015 & $2.76 \mathrm{E}-16$ \\
\hline $\mathrm{CO}$ & $16-15$ & 752.4 & 162.812 & $2.01 \mathrm{E}-15$ \\
\hline $\mathrm{U}$ & & & $161.808^{a}$ & $3.49 \mathrm{E}-17$ \\
\hline $\mathrm{C}^{+}$ & ${ }^{2} \mathrm{P}_{3 / 2}-{ }^{2} \mathrm{P}_{1 / 2}$ & 91.2 & 157.741 & $2.92 \mathrm{E}-16$ \\
\hline $\mathrm{H}_{2} \mathrm{O}$ & $3_{22}-3_{13}$ & 296.8 & 156.194 & $3.16 \mathrm{E}-16$ \\
\hline $\mathrm{CO}$ & $17-16$ & 846.3 & 153.267 & $2.15 \mathrm{E}-15$ \\
\hline $\mathrm{O}$ & ${ }^{3} \mathrm{P}_{0}-{ }^{3} \mathrm{P}_{1}$ & 326.6 & 145.525 & $8.38 \mathrm{E}-16$ \\
\hline $\mathrm{CO}$ & $18-17$ & 945.8 & 144.784 & 2.04E-15 \\
\hline $\mathrm{H}_{2} \mathrm{O}$ & $3_{13}-2_{02}$ & 204.7 & 138.527 & $9.49 \mathrm{E}-16$ \\
\hline
\end{tabular}

Notes. The horizontal line near $\sim 300 \mu \mathrm{m}$ shows the transition between the SPIRE SLW and SSW detector arrays (beams between 42" and $\left.17^{\prime \prime}\right)$. The line near $\sim 200 \mu \mathrm{m}$ marks the lines detected with PACS (spaxel size $\sim 9.4^{\prime \prime}$ ). Lines in the PACS ranges with detector leakage are not tabulated. Flux errors up to $\sim 30 \%$. Both the $o-\mathrm{H}_{2} \mathrm{O} 1_{01}$ and $p$ $\mathrm{H}_{2} \mathrm{O} 0_{00}$ ground-state levels are at $E_{\text {low }} / k=0 \mathrm{~K}$. ${ }^{(a)}$ Observed wavelength (otherwise rest wavelengths are tabulated).
Table A.1. continued.

\begin{tabular}{|c|c|c|c|c|}
\hline Species & Transition & $\begin{array}{l}E_{\text {up }} \\
(\mathrm{K})\end{array}$ & $\begin{array}{c}\lambda \\
(\mu \mathrm{m})\end{array}$ & $\begin{array}{l}\text { Line flux } \\
\left(\mathrm{W} \mathrm{m}^{-2}\right)\end{array}$ \\
\hline $\mathrm{CO}$ & $19-18$ & 1050.7 & 137.196 & $1.66 \mathrm{E}-15$ \\
\hline $\mathrm{H}_{2} \mathrm{O}$ & $5_{14}-5_{05}$ & 540.5 & 134.935 & $1.67 \mathrm{E}-16$ \\
\hline $\mathrm{U}$ & & & $132.982^{a}$ & $1.13 \mathrm{E}-16$ \\
\hline $\mathrm{U}$ & & & $132.672^{a}$ & $1.76 \mathrm{E}-16$ \\
\hline $\mathrm{H}_{2} \mathrm{O}$ & $4_{23}-4_{14}$ & 397.9 & 132.407 & $3.95 \mathrm{E}-16$ \\
\hline $\mathrm{CO}$ & $20-19$ & 1161.2 & 130.369 & $1.62 \mathrm{E}-15$ \\
\hline $\mathrm{H}_{2} \mathrm{O}$ & $4_{04}-3_{13}$ & 319.5 & 125.353 & $6.79 \mathrm{E}-16$ \\
\hline $\mathrm{CO}$ & $21-20$ & 1277.1 & 124.193 & $2.01 \mathrm{E}-15$ \\
\hline $\mathrm{OH}$ & ${ }^{2} \Pi_{3 / 2} J=5 / 2^{+}-3 / 2^{-}$ & 120.5 & 119.441 & $8.18 \mathrm{E}-16$ \\
\hline $\mathrm{OH}$ & ${ }^{2} \Pi_{3 / 2} J=5 / 2^{-}-3 / 2^{+}$ & 120.8 & 119.234 & $8.75 \mathrm{E}-16$ \\
\hline $\mathrm{CO}$ & $22-21$ & 1398.6 & 118.581 & $1.29 \mathrm{E}-15$ \\
\hline $\mathrm{H}_{2} \mathrm{O}$ & $4_{14}-3_{03}$ & 289.3 & 113.537 & $7.39 \mathrm{E}-17^{\dagger}$ \\
\hline $\mathrm{CO}$ & $23-22$ & 1525.5 & 113.458 & blended ${ }^{\dagger}$ \\
\hline $\mathrm{CO}$ & $24-23$ & 1657.9 & 108.763 & $1.08 \mathrm{E}-15$ \\
\hline $\mathrm{H}_{2} \mathrm{O}$ & $2_{21}-1_{10}$ & 159.9 & 108.073 & $1.35 \mathrm{E}-16$ \\
\hline $\mathrm{CO}$ & $27-26$ & 2087.9 & 96.773 & $9.06 \mathrm{E}-16$ \\
\hline $\mathrm{U}$ & & & $96.633^{a}$ & $6.14 \mathrm{E}-17$ \\
\hline $\mathrm{OH}$ & ${ }^{2} \Pi_{1 / 2}{ }^{2} \Pi_{3 / 2} J=3 / 2^{-}-5 / 2^{+}$ & 269.8 & 96.363 & $2.35 \mathrm{E}-16$ \\
\hline $\mathrm{OH}$ & ${ }^{2} \Pi_{1 / 2}-{ }^{2} \Pi_{3 / 2} J=3 / 2^{+}-5 / 2^{-}$ & 270.2 & 96.271 & $2.35 \mathrm{E}-16$ \\
\hline $\mathrm{H}_{2} \mathrm{O}$ & $5_{15}-4_{04}$ & 470.0 & 95.626 & $5.16 \mathrm{E}-16$ \\
\hline $\mathrm{H}_{2} \mathrm{O}$ & $4_{41}-4_{32}$ & 668.1 & 94.703 & $1.76 \mathrm{E}-16$ \\
\hline $\mathrm{CO}$ & $28-27$ & 2242.2 & 93.349 & $7.55 \mathrm{E}-16$ \\
\hline $\mathrm{U}$ & & & $90.574^{a}$ & $4.19 \mathrm{E}-17$ \\
\hline $\mathrm{U}$ & & & $90.434^{a}$ & $4.64 \mathrm{E}-18$ \\
\hline $\mathrm{CO}$ & $29-28$ & 2401.9 & 90.163 & $6.57 \mathrm{E}-16$ \\
\hline $\mathrm{H}_{2} \mathrm{O}$ & $3_{22}-2_{11}$ & 296.8 & 89.988 & $8.29 \mathrm{E}-16$ \\
\hline $\mathrm{CO}$ & $30-29$ & 2567.0 & 87.190 & $7.03 \mathrm{E}-16$ \\
\hline $\mathrm{U}$ & & & $86.944^{a}$ & $6.48 \mathrm{E}-17$ \\
\hline $\mathrm{H}_{2} \mathrm{O}$ & $7_{16}-7_{07}$ & 979.0 & 84.766 & $1.06 \mathrm{E}-16$ \\
\hline $\mathrm{OH}$ & ${ }^{2} \Pi_{3 / 2} J=7 / 2^{-}-5 / 2^{+}$ & 290.5 & 84.597 & $9.29 \mathrm{E}-16$ \\
\hline $\mathrm{CO}$ & $31-30$ & 2737.6 & 84.411 & $1.25 \mathrm{E}-15^{\dagger}$ \\
\hline $\mathrm{OH}$ & ${ }^{2} \Pi_{3 / 2} J=7 / 2^{+}-5 / 2^{-}$ & 291.2 & 84.420 & blended $^{\dagger}$ \\
\hline $\mathrm{H}_{2} \mathrm{O}$ & $6_{06}-5_{15}$ & 642.7 & 83.283 & $1.69 \mathrm{E}-16$ \\
\hline $\mathrm{H}_{2} \mathrm{O}$ & $6_{16}-5_{05}$ & 609.3 & 82.031 & $5.55 \mathrm{E}-16$ \\
\hline $\mathrm{CO}$ & $32-31$ & 2913.7 & 81.806 & $3.84 \mathrm{E}-16$ \\
\hline $\mathrm{U}$ & & & $81.013^{a}$ & $7.03 \mathrm{E}-17$ \\
\hline $\mathrm{CO}$ & $33-32$ & 3095.1 & 79.360 & $2.49 \mathrm{E}-16$ \\
\hline $\mathrm{OH}$ & ${ }^{2} \Pi_{1 / 2}-{ }^{2} \Pi_{3 / 2} J=1 / 2^{+}-3 / 2^{-}$ & 181.7 & 79.179 & blended $^{\dagger}$ \\
\hline $\mathrm{OH}$ & ${ }^{2} \Pi_{1 / 2}-{ }^{2} \Pi_{3 / 2} J=1 / 2^{-}-3 / 2^{+}$ & 181.9 & 79.116 & $9.97 \mathrm{E}-16^{\dagger}$ \\
\hline $\mathrm{H}_{2} \mathrm{O}$ & $4_{23}-3_{12}$ & 397.9 & 78.741 & $4.89 \mathrm{E}-17$ \\
\hline $\mathrm{CO}$ & $34-33$ & 3282.0 & 77.059 & $3.97 \mathrm{E}-16$ \\
\hline $\mathrm{H}_{2} \mathrm{O}$ & $3_{21}-2_{12}$ & 271.0 & 75.380 & 7.23E-16 \\
\hline $\mathrm{CO}$ & $35-34$ & 3474.3 & 74.890 & $3.53 \mathrm{E}-16$ \\
\hline $\mathrm{U}$ & & & $69.840^{a}$ & $5.75 \mathrm{E}-17$ \\
\hline $\mathrm{CO}$ & $38-37$ & 4083.5 & 69.074 & $1.57 \mathrm{E}-16$ \\
\hline $\mathrm{CO}$ & $39-38$ & 4297.3 & 67.336 & $1.28 \mathrm{E}-16$ \\
\hline $\mathrm{H}_{2} \mathrm{O}$ & $3_{03}-3_{03}$ & 376.4 & 67.269 & 7.77E-17 \\
\hline $\mathrm{H}_{2} \mathrm{O}$ & $3_{31}-2_{20}$ & 410.4 & 67.089 & $1.90 \mathrm{E}-16$ \\
\hline $\mathrm{H}_{2} \mathrm{O}$ & $3_{30}-2_{21}$ & 376.4 & 66.437 & $4.74 \mathrm{E}-16$ \\
\hline $\mathrm{H}_{2} \mathrm{O}$ & $7_{16}-6_{25}$ & 979.0 & 66.092 & $1.28 \mathrm{E}-16$ \\
\hline $\mathrm{CO}$ & 40-39 & 4516.6 & 65.686 & $8.33 \mathrm{E}-17$ \\
\hline $\mathrm{OH}$ & ${ }^{2} \Pi_{3 / 2} J=9 / 2^{+}-7 / 2^{-}$ & 511.0 & 65.279 & $4.45 \mathrm{E}-16$ \\
\hline $\mathrm{H}_{2} \mathrm{O}$ & $6_{25}-5_{14}$ & 761.3 & 65.165 & $1.99 \mathrm{E}-16$ \\
\hline $\mathrm{OH}$ & ${ }^{2} \Pi_{3 / 2} J=9 / 2^{-}-7 / 2^{+}$ & 512.1 & 65.132 & $4.11 \mathrm{E}-16$ \\
\hline $\mathrm{CO}$ & $41-40$ & 4741.2 & 64.117 & $1.23 \mathrm{E}-16$ \\
\hline $\mathrm{H}_{2} \mathrm{O}$ & $8_{08}-7_{17}$ & 1070.6 & 63.457 & $2.81 \mathrm{E}-17$ \\
\hline $\mathrm{H}_{2} \mathrm{O}$ & $8_{18}-7_{07}$ & 1036.5 & 63.322 & $4.00 \mathrm{E}-17$ \\
\hline $\mathrm{O}$ & ${ }^{3} \mathrm{P}_{1}-{ }^{3} \mathrm{P}_{2}$ & 227.7 & 63.183 & $8.34 \mathrm{E}-15$ \\
\hline $\mathrm{CO}$ & $42-41$ & 4971.1 & 62.624 & $6.82 \mathrm{E}-17$ \\
\hline $\mathrm{U}$ & & & $62.003^{a}$ & $9.05 \mathrm{E}-17$ \\
\hline $\mathrm{H}_{2} \mathrm{O}$ & $7_{26}-6_{15}$ & 1021.8 & 59.986 & $1.40 \mathrm{E}-16$ \\
\hline $\mathrm{H}_{2} \mathrm{O}$ & $4_{32}-3_{21}$ & 516.1 & 58.698 & $2.59 \mathrm{E}-16$ \\
\hline $\mathrm{H}_{2} \mathrm{O}$ & $4_{22}-3_{13}$ & 454.4 & 57.636 & $2.64 \mathrm{E}-16$ \\
\hline $\mathrm{H}_{2} \mathrm{O}$ & $4_{31}-3_{22}$ & 552.3 & 56.324 & $1.05 \mathrm{E}-16$ \\
\hline $\mathrm{U}$ & & & $55.481^{a}$ & $8.01 \mathrm{E}-17$ \\
\hline
\end{tabular}

Notes. (a) Observed wavelength (otherwise rest wavelengths are tabulated). 

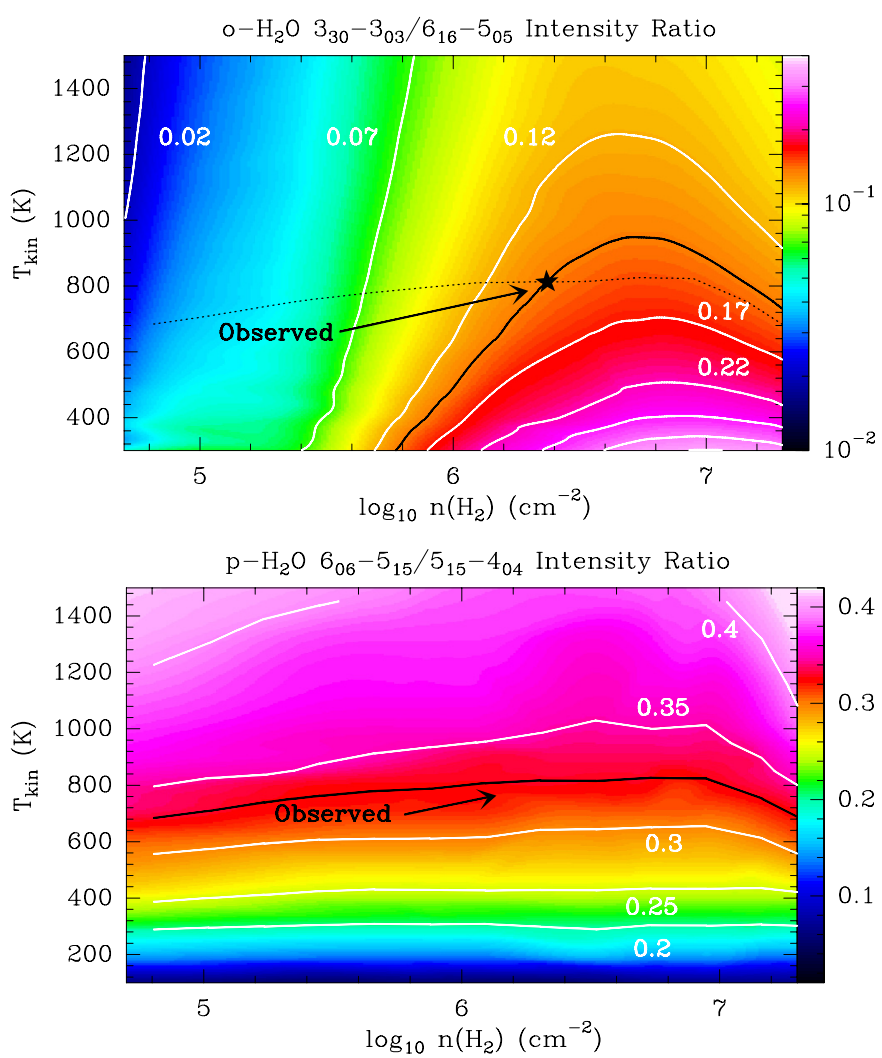

Fig. A.1. Grid of $\mathrm{H}_{2} \mathrm{O}$ LVG models for different gas temperatures and densities. Bottom: contour levels of the $p-\mathrm{H}_{2} \mathrm{O} 6_{06}-5_{15} / 5_{15}-4_{04}$ $(83.284 / 95.627 \mu \mathrm{m})$ line ratio for $N\left(p-\mathrm{H}_{2} \mathrm{O}\right)=10^{16} \mathrm{~cm}^{-2}$. Note the gas temperature dependence of this ratio. The black continuous curve shows the observed intensity line ratio of 0.33 . Top: contour levels of the $o-\mathrm{H}_{2} \mathrm{O} 3_{30}-3_{03} / 6_{15}-3_{05}(67.269 / 82.031 \mu \mathrm{m})$ ratio for $N\left(o-\mathrm{H}_{2} \mathrm{O}\right)=$ $3 \times 10^{16} \mathrm{~cm}^{-2}$. Note the gas density dependence of this ratio. The black continuous curve shows the observed intensity line ratio of 0.14 while the black dotted curve shows the observed $p-\mathrm{H}_{2} \mathrm{O} 6_{06}-5_{15} / 5_{15}-4_{04}$ ratio. The intersection of both curves is marked with a star (see text).

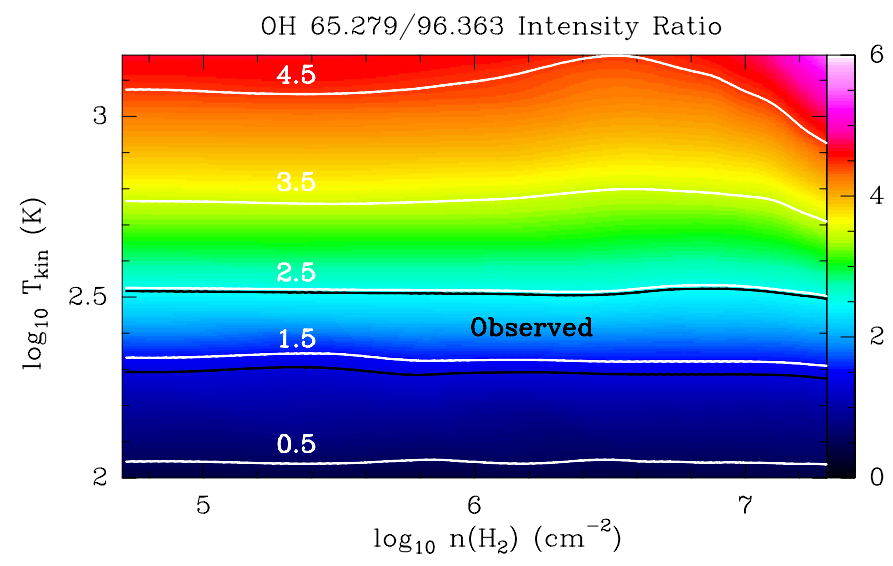

Fig. A.2. Grid of OH LVG models for different gas temperatures and densities. Contour levels of the $\mathrm{OH}^{2} \Pi_{3 / 2} J=9 / 2^{+}-7 / 2^{-} / \Pi_{1 / 2}{ }^{2} \Pi_{3 / 2}$ $J=3 / 2^{-}-5 / 2^{+}(65.279 / 96.363 \mu \mathrm{m})$ line ratio for $N(\mathrm{OH})=10^{16} \mathrm{~cm}^{-2}$. Note the gas temperature dependence of this ratio. The black continuous curves enclose the observed intensity line ratio with a $\sim 15 \%$ of relative flux calibration error.

Table A.2. Mid-IR lines detected with Spitzer/IRS towards the Class 0 protostar Serpens SMM1 and line fluxes (uncorrected for extinction) within a $7^{\prime \prime} \times 7^{\prime \prime}$ aperture.

\begin{tabular}{lcccc}
\hline \hline Species & Transition & $\begin{array}{c}E_{\text {up }} \\
(\mathrm{K})\end{array}$ & $\begin{array}{c}\lambda \\
(\mu \mathrm{m})\end{array}$ & $\begin{array}{c}\text { Line flux } \\
\left(\mathrm{W} \mathrm{m}^{-2}\right)\end{array}$ \\
\hline $\mathrm{Fe}^{+}$ & ${ }^{6} \mathrm{D}_{5 / 2}-{ }^{6} \mathrm{D}_{7 / 2}$ & 961 & 35.349 & $2.45 \mathrm{E}-17$ \\
$\mathrm{Si}^{+}$ & ${ }^{2} \mathrm{P}_{3 / 2}-{ }^{2} \mathrm{P}_{1 / 2}$ & 414 & 34.815 & $6.29 \mathrm{E}-17$ \\
$p-\mathrm{H}_{2}$ & $0-0 \mathrm{~S}(0) J=2-0$ & 509 & 28.219 & $<5.92 \mathrm{E}-18$ \\
$\mathrm{Fe}^{+}$ & ${ }^{6} \mathrm{D}_{7 / 2}-{ }^{6} \mathrm{D}_{9 / 2}$ & 554 & 25.988 & $8.12 \mathrm{E}-17$ \\
$\mathrm{~S}$ & ${ }^{3} \mathrm{P}_{1}-{ }^{3} \mathrm{P}_{2}$ & 570 & 25.249 & $3.24 \mathrm{E}-17$ \\
$\mathrm{Fe}^{+}$ & ${ }^{4} \mathrm{~F}_{5 / 2}-{ }^{4} \mathrm{~F}_{7 / 2}$ & 4087 & 24.519 & $4.46 \mathrm{E}-17$ \\
\hline $\mathrm{Fe}^{+}$ & ${ }^{4} \mathrm{~F}_{7 / 2}-{ }^{4} \mathrm{~F}_{9 / 2}$ & 3499 & 17.936 & $1.36 \mathrm{E}-17$ \\
$o-\mathrm{H}_{2}$ & $0-0 \mathrm{~S}(1) J=3-1$ & 1015 & 17.035 & $5.96 \mathrm{E}-19$ \\
$\mathrm{Ne}^{+}$ & ${ }^{2} \mathrm{P}_{1 / 2}-{ }^{2} \mathrm{P}_{3 / 2}$ & 1124 & 12.814 & $7.06 \mathrm{E}-18$ \\
$p-\mathrm{H}_{2}$ & $0-0 \mathrm{~S}(2) J=4-2$ & 1682 & 12.279 & $2.42 \mathrm{E}-18$ \\
\hline
\end{tabular}

Notes. The horizontal line shows the transition between the LH and $\mathrm{SH}$ modules. Intensity errors up to $\sim 25 \%$. 
J. R. Goicoechea et al.: The complete spectrum of the Class 0 protostar Serpens SMM1

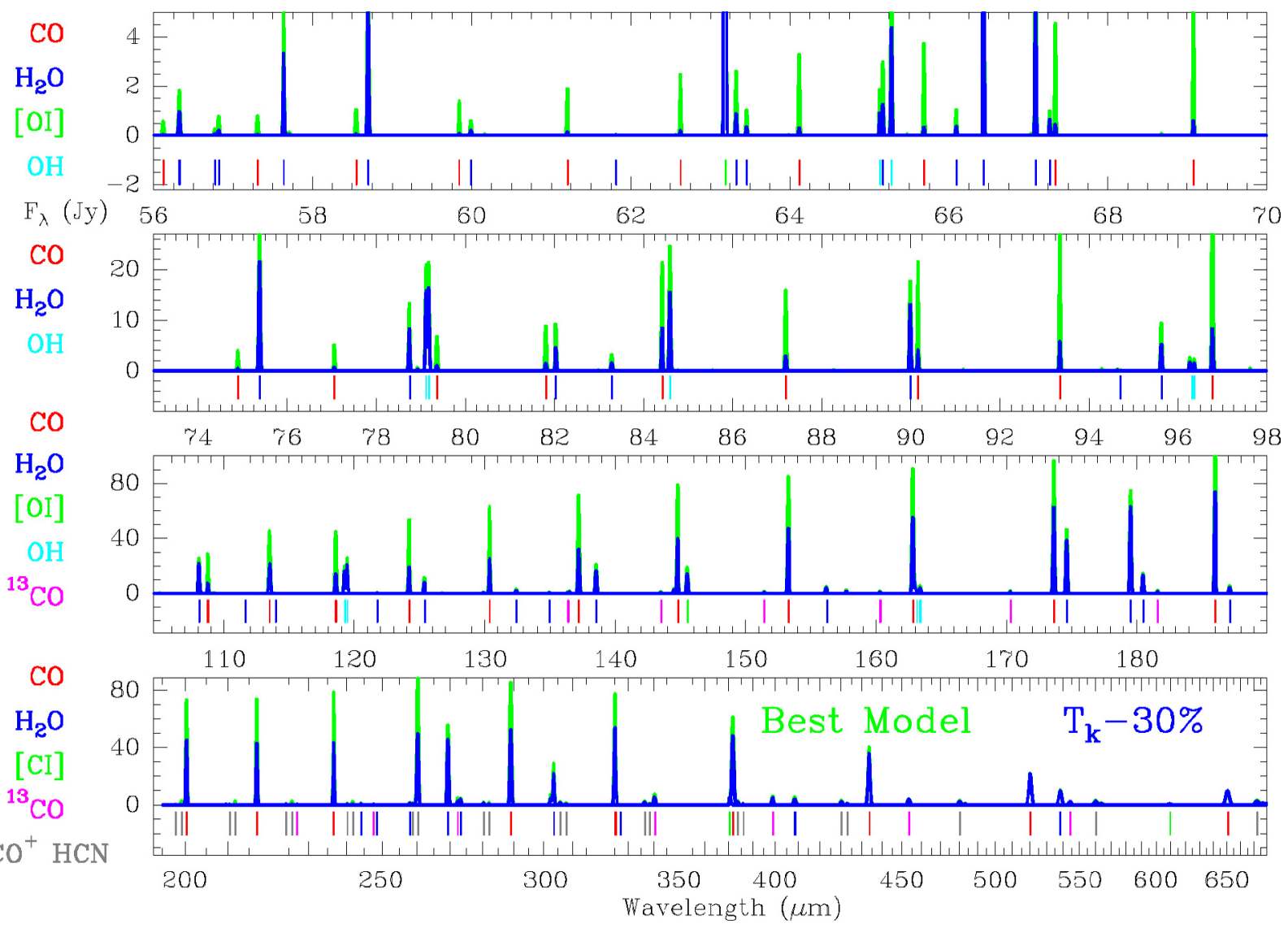

Fig. A.3. Best model discussed in the text (green curves) and a model where $T_{\mathrm{k}}$ is decreased by $30 \%$ in all components (blue curves).

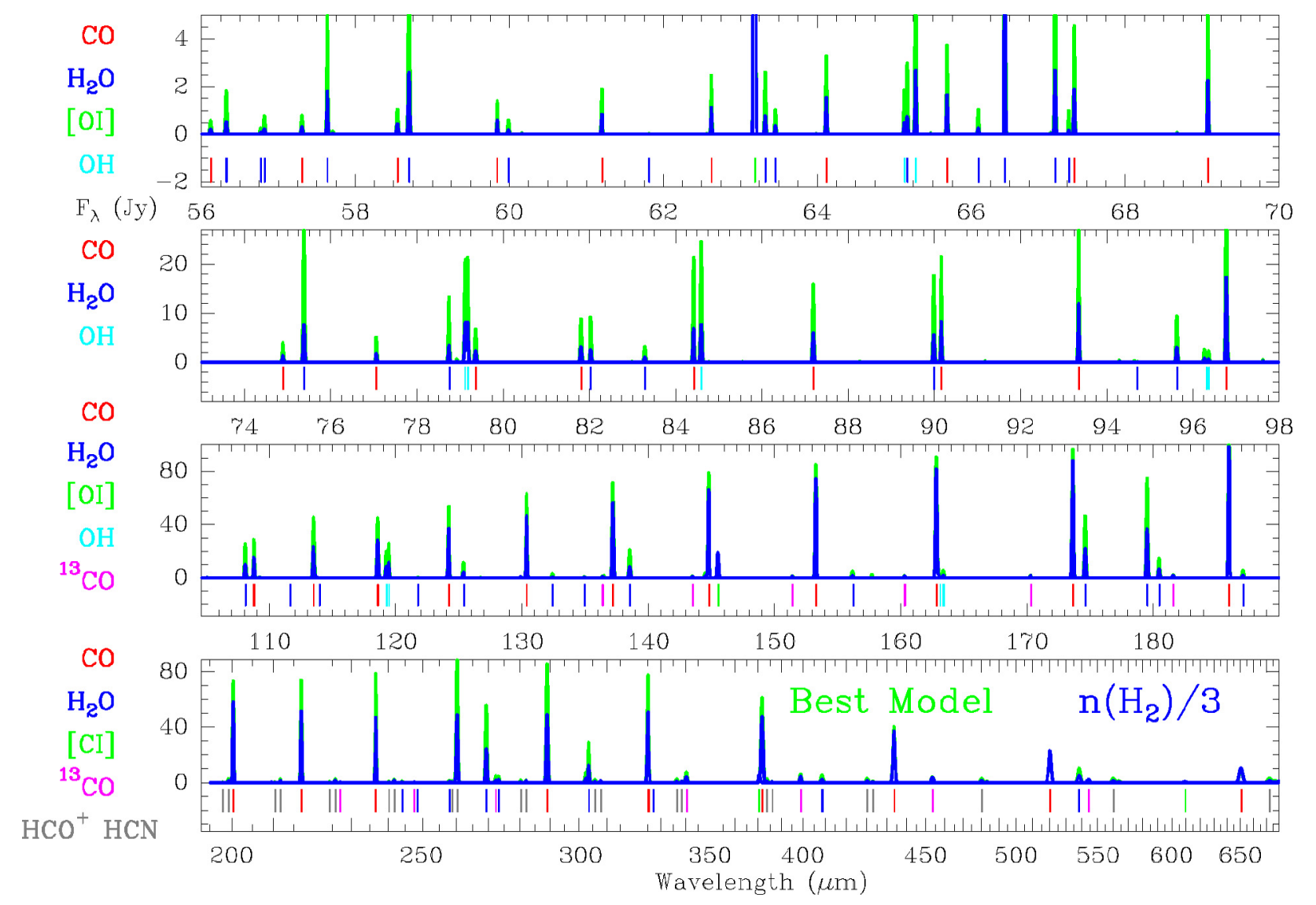

Fig. A.4. Best model discussed in the text (green curves) and a model where $n\left(\mathrm{H}_{2}\right)$ is decreased by a factor 3 in all components (blue curves). 